\title{
Asymmetric Intermolecular C-H Functionalization Of Benzyl Silyl Ethers Mediated By Chiral Auxiliary-Based Aryldiazoacetates and Chiral Dirhodium Catalysts
}

Huw M. L. Davies*, Simon J. Hedley and Brooks R. Bohall

Department of Chemistry, University at Buffalo, The State University of New York, Buffalo, New York 14260-3000.

hdavies@acsu.buffalo.edu

\section{Supporting Information}

Table Of Contents

General Considerations

S2

Determination of Relative Stereochemistry Of C-H Functionalization Reaction Products

Experimental Procedures And Characterisation Data

${ }^{1} \mathrm{H}$ NMR Spectrum of $\mathbf{6 b}$

S4-S33

${ }^{1} \mathrm{H}$ NMR Spectrum of $\mathbf{1 1}$ S34

${ }^{1} \mathrm{H}$ NMR Spectrum of $\mathbf{1 3 e}$

S35

${ }^{1} \mathrm{H} \quad \mathrm{NMR} \quad$ Spectrum of $(S)$ - Ethyl 2-(2-(4S36 bromophenyl)acetoxy)propanoate

${ }^{1} \mathrm{H}$ NMR Spectrum of $(S)$-2-[2-(4-Bromo-phenyl)-2-diazo-acetoxy]propionic acid ethyl ester

${ }^{1}$ H NMR Spectrum of $(S)$-Ethyl 2-(2-p-tolylacetoxy)propanoate

${ }^{1} \mathrm{H}$ NMR Spectrum of $(S)$-2-[2-Diazo-2-(4-methoxy-phenyl)acetoxy]-propionic acid ethyl ester

${ }^{1}$ H NMR Spectrum of $(S)$-2-[2-Diazo-2-(4-trifluoromethyl-phenyl)acetoxy]-propionic acid ethyl ester

${ }^{1} \mathrm{H}$ NMR Spectrum of $\mathbf{1 5}$

S37

${ }^{1} \mathrm{H}$ NMR Spectrum of $\mathbf{1 6}$

S38

${ }^{1} \mathrm{H}$ NMR Spectrum of $\mathbf{2 5}$

S39

S40

${ }^{13} \mathrm{C}$ NMR Spectrum of $\mathbf{2 5}$

S41

S42

S43

S44

X-Ray Structure of (1R,2S)-2-(4-Bromophenyl)-1-(4methoxyphenyl)propane-1,3-diol 23 


\section{General Considerations}

All reactions were conducted in flame-dried glassware under an inert atmosphere of dry argon. All reagents were used as received from commercial suppliers unless otherwise stated. Acetonitrile, dichloromethane, hexane, tetrahydrofuran and toluene were purified by passage through a bed of activated alumina. 2,2-Dimethylbutane was distilled from sodium metal after passing through a pad of activated silica gel. All solvents used for $\mathrm{C}-\mathrm{H}$ functionalization reactions were degassed by bubbling argon through the solvent for 15 min prior to use. Flash chromatography was performed on silica gel (32-63D $60 \AA$ ) according to the method of W.C. Still. ${ }^{1}$ Thin layer chromatography (TLC) was performed on aluminium backed plates pre-coated with silica $(0.25 \mathrm{~mm}, 60 \mathrm{~F}-254)$ which were developed using standard visualizing agents: UV fluorescence (254 and $366 \mathrm{~nm}$ ), phosphomolybdic acid / $\Delta$. Melting points were determined using an electrothermal melting point apparatus and are uncorrected. Optical rotations were measured on a digital polarimeter at $25{ }^{\circ} \mathrm{C} .{ }^{1} \mathrm{H}$ NMR spectra were recorded on a Nuclear Magnetic Resonance spectrometer at 500, 400, or $300 \mathrm{MHz}$, and ${ }^{13} \mathrm{C}$ NMR spectra were recorded on a Nuclear Magnetic Resonance spectrometer at $125 \mathrm{MHz}$ or $75 \mathrm{MHz}$, calibrated by using residual undeuterated solvent as an internal standard. The following abbreviations apply: (b) broad. (s) singlet, (d) doublet, (t) triplet, (q) quartet, (m) multiplet, (dd) double doublet, etc. Chemical shifts are given in ppm. $J$ values are recorded in $\mathrm{Hz}$ and are rounded to the nearest half-integer. Infrared (IR) spectra were determined using a FTIR spectrometer. Low resolution mass spectra $(\mathrm{m} / \mathrm{z})$ and high resolution mass spectra (HRMS) were obtained from an external service provider. Elemental analyses were determined by an external service provider. Diastereomeric ratios were determined by values derived from the $500 \mathrm{MHz}{ }^{1} \mathrm{H}$ NMR spectra of the crude reaction mixture. Enantiomeric excess was determined by high performance liquid chromatography (HPLC) using chiral analytical columns with 2-propanol in hexane as eluant.

The known compounds tert-butyl-(4-methoxybenzyloxy)-dimethylsilane $\mathbf{6 a},{ }^{2}$ benzyloxytert-butyl-dimethyl-silane $\mathbf{6 c},{ }^{3}$ (4-methoxybenzyloxy)-trimethylsilane, ${ }^{4}$ triisopropyl-(4-

\footnotetext{
${ }^{1}$ Still, W. C.; Kahn, M.; Mitra, M. J. Org. Chem. 1978, 43, 2923.

${ }^{2}$ Kennedy-Smith, J. J.; Nolin, K. A.; Gunterman, H. P.; Toste, F. D. J. Am. Chem. Soc. 2003, 125, 4056.

${ }^{3}$ Balduzzi, S.; Brook, M. A. Tetrahedron 2000, 56, 1617.

${ }^{4}$ Nishiyama, Y.; Kajimoto, H.; Kotani, K.; Nishida, T.; Sonoda, N. J. Org. Chem. 2002, $67,5696$.
} 
methoxybenzyloxy)-silane, ${ }^{5}$ methyl phenyldiazoacetate $7,{ }^{6}$ tert-butyl phenyldiazoacetate 9, ${ }^{7} \mathrm{Rh}_{2}(R$-DOSP $){ }_{4}{ }^{7} \mathrm{Rh}_{2}(S \text {-DOSP })_{4} \mathbf{2}^{7}$, para-acetaminobenzenesulfonyl azide, ${ }^{8} \mathrm{Rh}_{2}(S$ biTISP $)_{2} 3^{9}$, catalyst $\mathbf{1 8},{ }^{10} \mathrm{Rh}_{2}(S \text {-PTTL })_{4} \mathbf{5},{ }^{11} \mathrm{Rh}_{2}(S \text {-NTTL })_{4} \mathbf{1 9},{ }^{12} \mathrm{Rh}_{2}(S \text {-BNP })_{4} \mathbf{2 0},{ }^{13}$ methyl para-bromophenyldiazoacetate, ${ }^{14}$ methyl para-methoxyphenyldiazoacetate ${ }^{14}$ and silyl ether $\mathbf{2 4}{ }^{15}$ were prepared by literature procedures and the spectral data showed good agreement with the literature data.

\section{Determination of Relative Stereochemistry of C-H Functionalization Reaction Products}

The diastereomeric excess (\%de) was determined by analysis of the crude proton NMR spectrum: applying our structural analysis model, if the products of $\mathrm{C}-\mathrm{H}$ functionalization exist in well-defined conformations, the stereochemistry can be assigned by distinctive proton NMR chemical shift differences between the two diastereoisomers. ${ }^{16}$ The $\mathrm{C}-\mathrm{H}$ Functionalization products 8a-e all have large coupling constants $(J=8.5$ to $11.5 \mathrm{~Hz}$ ), and are thus expected to exist in a conformation where the hydrogen atoms are arranged antiperiplanar. Observing the relevant Newman projections of the two diastereoisomers, with respect to the tert-butyldimethylsilyl groups, the syn isomer 8a should be expected to be shielded by the aromatic ring, whilst the anti isomer 8a should be comparatively deshielded (Figure S1).

\footnotetext{
${ }^{5}$ Sebhat, M. K.; Tan, Y.-L.; Widdowson, D. A.; Wilhelm, R.; White, A. J. P.; Williams, D. J. Tetrahedron 2000, 56, 6121.

${ }^{6}$ Takamura, N.; Mizoguchi, T.; Koga, K.; Yamada, S. Tetrahedron 1975, 31, 227.

${ }^{7}$ Davies, H. M. L.; Bruzinski, P. R.; Lake, D. H.; Kong, N.; Fall, M. J. J. Am. Chem. Soc. 1996, $118,6897$.

${ }^{8}$ Baum, J. S.; Shook, D. A.; Davies, H. M. L.; Smith, H. D. Synth. Commun. 1987, 17, 1709.

${ }^{9}$ Davies, H. M. L.; Panaro, S. Tetrahedron Lett. 1999, 40, 5287.

${ }^{10}$ Davies, H. M. L.; Kong, N. Tetrahedron Lett. 1997, 38, 4203.

${ }^{11}$ Tetrahedron Lett. 1993, 34, 5109.

${ }^{12}$ Müeller, P.; Bernardinelli, G.; Allenbach, Y. F.; Ferri, M., Flack, H. D. Org. Lett. 2004, 6, 1725.

${ }^{13}$ Pirrung, M. C.; Zhang, J. Tetrahedron Lett. 1992, 33, 5987.

${ }^{14}$ Davies, H. M. L.; Hansen, T.; Churchill, M. R. J. Am. Chem. Soc. 2000, 122, 3063.

${ }^{15}$ Wang, J.-X.; Tu, Y.; Frohn, M.; Zhang, J._R.; Shi, Y. J. Am. Chem. Soc. 1997, 119, 11224.

${ }^{16}$ Davies, H. M. L.; Ren, P. Tetrahedron Lett. 2001, 42, 3149.
} 


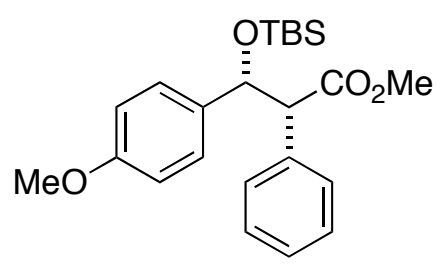

$\operatorname{syn} 8 \mathbf{a}$

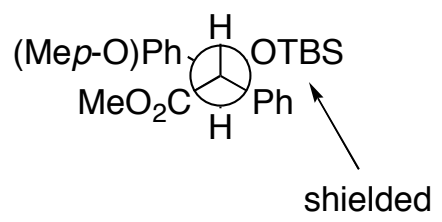<smiles>COc1ccc([C@H]([OH2+])C(c2ccccc2)c2ccccc2)cc1</smiles>

anti 8a

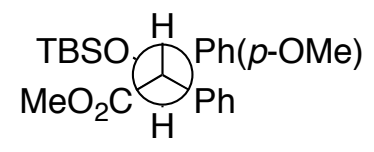

Figure S1. Relative Stereochemistry Of syn $\mathbf{8 a}$ and anti $\mathbf{8 a}$.

The chemical shift for the methyl groups of the TBS group of $\mathbf{8 a}$ come into resonance at _-0.37 and _- 0.39 , and the tert-butyl group at _ 0.61 for the major isomer; and at 0.05 and -0.26 for the methyl groups and 0.84 for the tert-butyl groups of the minor isomer, therefore the major isomer is the syn product 8a. Additionally, the relative stereochemistry was confirmed from the x-ray data of diol 23.'

\section{Experimental Procedures and Characterisation Data} tert-Butyl-dimethyl-(4-methyl-benzyloxy)-silane (6b)<smiles>Cc1ccc(CO[Se-](C)(C)C)cc1</smiles>

A solution of 4-methylbenzyl alcohol (5.00 g, $40.90 \mathrm{mmol}, 1$ eq.) and tertbutyldimethylsilyl chloride (6.48 g, $43.00 \mathrm{mmol}, 1.05$ eq.) in $\mathrm{CH}_{2} \mathrm{Cl}_{2}$ (250 mL) was stirred until a homogeneous solution was obtained. Imidazole (3.06 g, 44.90 mmol, 1.1 eq.) was added in one portion, and the opaque milky-white suspension was stirred for $3 \mathrm{~h}$, and then pentane $(50 \mathrm{~mL})$ was added, and stirred for a further $10 \mathrm{~min}$. The suspension was then filtered through a plug of silica and concentrated. The residue was purified by flash chromatography on silica gel using 20:1 hexane/Et ${ }_{2} \mathrm{O}$ to provide a colorless oil, 8.17 g (84\%). ${ }^{1} \mathrm{H}$ NMR (500 MHz, $\left.\mathrm{CDCl}_{3}\right)$ :_ 7.22 (d, 2H, J=8.0 Hz), 7.15 (d, 2H, J=8.0 Hz), $4.71(\mathrm{~s}, 2 \mathrm{H}), 2.35$ (s, 3H), 0.95 (s, 9H), $0.10(\mathrm{~s}, 6 \mathrm{H}) .{ }^{13} \mathrm{C}$ NMR (500 MHz, $\left.\mathrm{CDCl}_{3}\right)$ : _ 138.4, 136.4, 128.9, 126.1, 64.9, 26.0, 21.1, 18.4, -5.2. IR (film): 2956, 2930, 2858, 
$1517,1471,1257,1091,838 \mathrm{~cm}^{-1} . \mathrm{m} / z 236\left(\mathrm{M}^{+}, 4 \%\right), 221(8 \%), 179(100 \%), 149(24 \%)$, 105 (93\%). HRMS Calcd. for $\mathrm{C}_{14} \mathrm{H}_{24} \mathrm{OSi}\left(\mathrm{M}^{+}\right)$: 236.1591. Found: 236.1585.

tert-Butyl-(4-chloro-benzyloxy)-dimethyl-silane (6d)

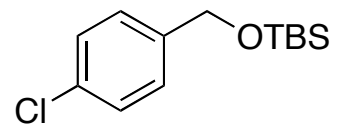

A solution of 4-chlorobenzyl alcohol (5.00 g, $35.06 \mathrm{mmol}, 1$ eq.) and tertbutyldimethylsilyl chloride (5.55 g, $36.82 \mathrm{mmol}, 1.05$ eq.) in $\mathrm{CH}_{2} \mathrm{Cl}_{2}$ (250 mL) was stirred until a homogeneous solution was obtained. Imidazole $(2.63 \mathrm{~g}, 38.57 \mathrm{mmol}, 1.1$ eq.) was added in one portion, and the opaque milky-white suspension was stirred for $3 \mathrm{~h}$, and then pentane $(50 \mathrm{~mL})$ was added, and stirred for a further $10 \mathrm{~min}$. The suspension was then filtered through a plug of silica and concentrated. The residue was purified by flash chromatography on silica gel using 20:1 hexane/Et ${ }_{2} \mathrm{O}$ to provide a colorless oil, 8.79 g (98\%). ${ }^{1} \mathrm{H}$ NMR (500 MHz, $\left.\mathrm{CDCl}_{3}\right)$ : _7.28-7.26 (m, 2H), 7.21-7.17 (m, 2H), 4.73 (s, 2H), 0.96 (s, 9H), 0.12 (s, 6H). ${ }^{13} \mathrm{C}$ NMR (125 MHz, $\left.\mathrm{CDCl}_{3}\right)$ : ${ }_{1}$ 140.1, 132.7, 128.6, 127.6, 64.5, 26.2, 18.6, -5.0. IR $\left(\mathrm{CH}_{2} \mathrm{Cl}_{2}\right): 2955,2931,2858,1492,1257,1088 \mathrm{~cm}^{-1} . \mathrm{m} / z$ (ESI): $256\left(\mathrm{M}^{+}, 1 \%\right), 199$ (100\%), 169 (42\%), 125 (91\%). Anal. calcd. for $\mathrm{C}_{13} \mathrm{H}_{21} \mathrm{OClSi}$ : C, 60.79; H, 8.24. Found: C, 60.65; H, 8.21.

tert-Butyl-dimethyl-(4-trifluoromethyl-benzyloxy)-silane (6e)<smiles>FC(F)(F)c1ccc(CO[SbH3])cc1</smiles>

A solution of 4-(trifluoromethyl)-benzyl alcohol (5.00 g, $28.40 \mathrm{mmol}, 1$ eq.) and tertbutyldimethylsilyl chloride (4.49 g, $29.80 \mathrm{mmol}, 1.05$ eq.) in $\mathrm{CH}_{2} \mathrm{Cl}_{2}$ (250 mL) was stirred until a homogeneous solution was obtained. Imidazole (2.13 g, $31.3 \mathrm{mmol}, 1.1$ eq.) was added in one portion, and the opaque milky-white suspension was stirred for $3 \mathrm{~h}$, and then pentane $(50 \mathrm{~mL})$ was added, and stirred for a further $10 \mathrm{~min}$. The suspension was then filtered through a plug of silica and concentrated. The residue was purified by flash chromatography on silica gel using $20: 1$ hexane/Et ${ }_{2} \mathrm{O}$ to provide a colorless oil, 7.03 g (85\%). ${ }^{1} \mathrm{H}$ NMR (500 MHz, $\left.\mathrm{CDCl}_{3}\right)$ : 7.60 (d, 2H, J=8.0 Hz), 7.44 (d, 2H, J=8.0 Hz), $4.80(\mathrm{~s}, 2 \mathrm{H}), 0.96(\mathrm{~s}, 9 \mathrm{H}), 0.12(\mathrm{~s}, 6 \mathrm{H}) .{ }^{13} \mathrm{C} \mathrm{NMR}\left(125 \mathrm{MHz}, \mathrm{CDCl}_{3}\right)$ : $145.5,129.1$ (q, $J=31.5 \mathrm{~Hz}$ ), 126.0, 125.1 (q, $J=4.0 \mathrm{~Hz}), 124.3$ (q, $J=271.5 \mathrm{~Hz}), 64.3,25.9,18.4$, -5.3. IR 
$\left(\mathrm{CH}_{2} \mathrm{Cl}_{2}\right): 2955,2930,2860,1473,1327,1127,1095 \mathrm{~cm}^{-1} . \mathrm{m} / \mathrm{z}$ (ESI): $290\left(\mathrm{M}^{+}, 4 \%\right), 271$ (100\%). Anal calcd. for $\mathrm{C}_{14} \mathrm{H}_{21} \mathrm{OF}_{3} \mathrm{Si}: \mathrm{C}, 57.90 ; \mathrm{H}, 7.29$. Found: C, 57.98; H, 7.37.

(2R,3R)-Methyl 3-(tert-butyldimethylsilyloxy)-3-(4-methoxyphenyl)-2phenylpropanoate (8a)

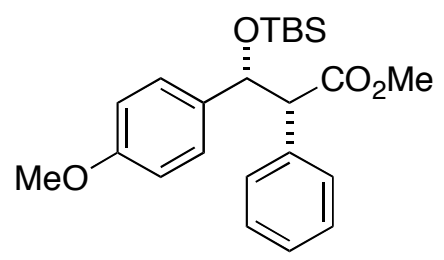

Procdure A: A solution of methyl phenyldiazoacetate 7 (352 mg, $2.00 \mathrm{mmol}, 2.00$ eq.) in 2,2-dimethylbutane $(8 \mathrm{~mL})$ was added to a solution of $\mathrm{Rh}_{2}(R \text {-DOSP })_{4}(19 \mathrm{mg}, 0.01$ mmol, 0.01 eq.) and compound $6 \mathbf{a}(252 \mathrm{mg}, 1.00 \mathrm{mmol}, 1.00$ eq.) in 2,2-dimethylbutane $(1 \mathrm{~mL}$ ) via syringe pump at a rate of $4 \mathrm{ml} / \mathrm{h}$. After addition was complete, the reaction mixture was stirred for $1 \mathrm{~h}$, and then concentrated in vacuo. Diastereoselectivity ( $88 \% \mathrm{de}$ ) was determined by ${ }^{1} \mathrm{H}$ NMR of the crude reaction mixture, and the products purified by flash chromatography on silica gel using $18: 1$ hexane/ $\mathrm{Et}_{2} \mathrm{O}$ as eluant to provide the anti isomer as a white solid, $15 \mathrm{mg}$; a mixture of anti and syn isomer as a white solid, $41 \mathrm{mg}$; and pure syn isomer as a white solid, $283 \mathrm{mg}$ (total yield: 85\%).

Procdure B: A solution of methyl phenyldiazoacetate 7 (176 mg, $1.00 \mathrm{mmol}, 2.00$ eq.) in 2,2-dimethylbutane $(6 \mathrm{~mL})$ was added to a solution of $\mathrm{Rh}_{2}(S \text {-PTTL })_{4}(6 \mathrm{mg}, 0.005 \mathrm{mmol}$, 0.01 eq.) and compound $6 \mathbf{6}(126 \mathrm{mg}, 0.50 \mathrm{mmol}, 1.00$ eq.) and 2,2-dimethylbutane (3 $\mathrm{mL}$ ) under reflux via syringe pump at a rate of $2 \mathrm{ml} / \mathrm{h}$. After addition was complete, the reaction mixture was stirred at $50{ }^{\circ} \mathrm{C}$ for $30 \mathrm{~min}$, and then allowed to cool to room temperature and concentrated in vacuo. Diastereoselectivity ( $89 \%$ de) was determined by ${ }^{1} \mathrm{H}$ NMR of the crude reaction mixture, and the products purified by flash chromatography on silica gel using $18: 1$ hexane/ $\mathrm{Et}_{2} \mathrm{O}$ as eluant to provide a mixture of anti and syn isomers as a white solid, $9 \mathrm{mg}$; and pure syn isomer as a white solid, $147 \mathrm{mg}$ (total yield: $78 \%$ ). Mp 62-64 ${ }^{\circ} \mathrm{C} .[\alpha]_{\mathrm{D}}+75.9$ (ee 91\%) (c 1.16, $\left.\mathrm{CHCl}_{3}\right) .{ }^{1} \mathrm{H}$ NMR (500 $\left.\mathrm{MHz}, \mathrm{CDCl}_{3}\right)$ : $7.42(\mathrm{~d}, 2 \mathrm{H}, J=7.5 \mathrm{~Hz}), 7.34-7.21(\mathrm{~m}, 5 \mathrm{H}), 6.81(\mathrm{~d}, 2 \mathrm{H}, J=9.0 \mathrm{~Hz}), 5.06$ (d, 1H, $J=9.0 \mathrm{~Hz}$ ), 3.79 (s, 3H), 3.78 (d, 1H, $J=9.0 \mathrm{~Hz}$ ), 3.43 (s, 3H), 0.61 (s, 9H), -0.37 (s, 3H), -0.39 (s, 3H). ${ }^{13} \mathrm{C}$ NMR (125 MHz, $\left.\mathrm{CDCl}_{3}\right)$ :_172.2, 158.9, 136.6, 135.0, 129.3, 128.1 (2C), 127.3, 113.2, 76.7, 61.7, 55.1, 51.6, 25.4, 17.8, -5.0, -5.8. IR $\left(\mathrm{CHCl}_{3}\right): 2953$, 
2929, 2856, 1736, 1612, $1512 \mathrm{~cm}^{-1} \cdot \mathrm{m} / z$ (ESI): $423\left(\mathrm{MNa}^{+}, 65 \%\right), 270(14 \%), 269$ (100\%). Anal. calcd. for $\mathrm{C}_{23} \mathrm{H}_{32} \mathrm{O}_{4} \mathrm{Si}: \mathrm{C}, 68.96: \mathrm{H}, 8.05$. Found: C, 68.94; H, 8.08. HPLC analysis: $91 \%$ ee (Chiralcel OD-H, $1 \% i-\mathrm{PrOH}$ in hexane, $1.0 \mathrm{ml} / \mathrm{min}, \lambda=254 \mathrm{~nm}, t_{\mathrm{R}}=4.8$ min, minor; $t_{\mathrm{R}}=5.7$ min, major).

(2R,3R)-Methyl 3-(tert-butyldimethylsilyloxy)-2-phenyl-3-p-tolylpropanoate (8b)

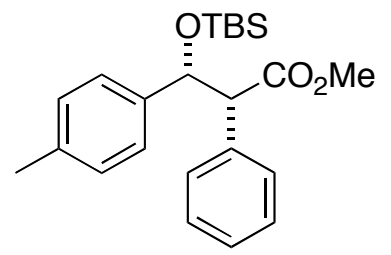

Procdure A: A solution of methyl phenyldiazoacetate 7 (352 mg, $2.00 \mathrm{mmol}, 2.00$ eq.) in 2,2-dimethylbutane $(8 \mathrm{~mL})$ was added to a solution of $\mathrm{Rh}_{2}(R \text {-DOSP })_{4}(19 \mathrm{mg}, 0.01$ mmol, 0.01 eq.) and compound $\mathbf{6 b}(236 \mathrm{mg}, 1.00 \mathrm{mmol}, 1.00$ eq.) in 2,2-dimethylbutane $(1 \mathrm{~mL}$ ) via syringe pump at a rate of $4 \mathrm{ml} / \mathrm{h}$. After addition was complete, the reaction mixture was stirred for $1 \mathrm{~h}$, and then concentrated in vacuo. Diastereoselectivity ( $91 \% \mathrm{de})$ was determined by ${ }^{1} \mathrm{H}$ NMR of the crude reaction mixture, and the products purified by flash chromatography on silica gel using $18: 1$ hexane/ $\mathrm{Et}_{2} \mathrm{O}$ to isolate the anti isomer as a white solid, $13 \mathrm{mg}$; and the syn isomer as a white solid, $310 \mathrm{mg}$ (total yield: 84\%).

Procdure B: A solution of methyl phenyldiazoacetate 7 (176 mg, $1.00 \mathrm{mmol}, 2.00$ eq.) in 2,2-dimethylbutane $(6 \mathrm{~mL})$ was added to a solution of $\mathrm{Rh}_{2}(S \text {-PTTL })_{4}(6 \mathrm{mg}, 0.005 \mathrm{mmol}$, 0.01 eq.) and compound $\mathbf{6 b}$ (118 $\mathrm{mg}, 0.50 \mathrm{mmol}, 1.00$ eq.) and 2,2-dimethylbutane (3 $\mathrm{mL}$ ) under reflux via syringe pump at a rate of $2 \mathrm{ml} / \mathrm{h}$. After addition was complete, the reaction mixture was stirred at $50{ }^{\circ} \mathrm{C}$ for $30 \mathrm{~min}$, and then allowed to cool to room temperature and concentrated in vacuo. Diastereoselectivity ( $94 \%$ de) was determined by ${ }^{1} \mathrm{H}$ NMR of the crude reaction mixture, and the products purified by flash chromatography on silica gel using 18:1 hexane/ $\mathrm{Et}_{2} \mathrm{O}$ as eluant to provide a mixture of anti and syn isomers as a white solid, $29 \mathrm{mg}$; and pure syn isomer as a white solid, 132 mg (total yield: 84\%). Mp 55-57 ${ }^{\circ} \mathrm{C}$. [_] $]_{\mathrm{D}}+79.7$ (ee 98\%) (c 1.53, $\left.\mathrm{CHCl}_{3}\right) .{ }^{1} \mathrm{H}$ NMR (500 $\left.\mathrm{MHz}, \mathrm{CDCl}_{3}\right)$ : 7.44 (d, 2H, J=7.0 Hz), 7.34-7.23 (m, 5H), 7.08 (d, 2H, J=8.0 Hz), 5.09 (d, 1H, J=9.0 Hz), 3.79 (d, 1H, J=9.0 Hz), 3.43 (s, 3H), 2.32 (s, 3H), 0.62 (s, 9H), -0.37 (s, 3H), -0.38 (s, 3H). ${ }^{13} \mathrm{C}$ NMR (125 MHz, $\mathrm{CDCl}_{3}$ ):_172.4, 140.1, 137.4, 136.9, 129.7, $128.9,128.4,127.6,127.1,77.2,62.0,51.9,25.8,21.5,18.1,-4.7,-5.5 . \mathrm{IR}\left(\mathrm{CHCl}_{3}\right)$ : 
2952, 2928, 2856, 2887, 1736, $1087 \mathrm{~cm}^{-1} . \mathrm{m} / \mathrm{z}$ (ESI): $384\left(\mathrm{M}^{+}, 9 \%\right), 284$ (43\%), 253 (100\%), 221 (10\%). Anal. calcd. for $\mathrm{C}_{23} \mathrm{H}_{32} \mathrm{O}_{3} \mathrm{Si}$ : C, 71.83: H, 8.39. Found: C, 71.73; H, 8.42. HPLC analysis: $98 \%$ ee (Chiralcel OD-H, $1 \% i$-PrOH in hexane, $1.0 \mathrm{ml} / \mathrm{min}, \quad=254$ $\mathrm{nm}, t_{\mathrm{R}}=3.7 \mathrm{~min}$, minor; $t_{\mathrm{R}}=4.8$ min, major).

(2R,3R)-Methyl 3-(tert-butyldimethylsilyloxy)-2,3-diphenylpropanoate (8c)<smiles>CO[C@@H](c1ccccc1)[C@H](OC)c1ccccc1</smiles>

Procdure A: A solution of methyl phenyldiazoacetate 7 (352 mg, $2.00 \mathrm{mmol}, 2.00$ eq.) in 2,2-dimethylbutane $(8 \mathrm{~mL})$ was added to a solution of $\mathrm{Rh}_{2}(R \text {-DOSP })_{4}(19 \mathrm{mg}, 0.01$ mmol, 0.01 eq.) and compound $6 \mathbf{c}$ (223 mg, $1.00 \mathrm{mmol}, 1.00$ eq.) in 2,2-dimethylbutane $(1 \mathrm{~mL}$ ) via syringe pump at a rate of $4 \mathrm{ml} / \mathrm{h}$. After addition was complete, the reaction mixture was stirred for $1 \mathrm{~h}$, and then concentrated in vacuo. Diastereoselectivity ( $91 \% \mathrm{de}$ ) was determined by ${ }^{1} \mathrm{H}$ NMR of the crude reaction mixture, and the products purified by flash chromatography on silica gel using $18: 1$ hexane/ $\mathrm{Et}_{2} \mathrm{O}$ as eluant to isolate the a mixture of anti and syn isomers as a white solid, $12 \mathrm{mg}$; and pure syn isomer as a white solid, $316 \mathrm{mg}$ (total yield: 83\%).

Procdure B: A solution of methyl phenyldiazoacetate 7 (176 mg, $1.00 \mathrm{mmol}, 2.00$ eq.) in 2,2-dimethylbutane $(6 \mathrm{~mL})$ was added to a solution of $\mathrm{Rh}_{2}(S \text {-PTTL })_{4}(6 \mathrm{mg}, 0.005 \mathrm{mmol}$, 0.01 eq.) and compound $\mathbf{6 c}(112 \mathrm{mg}, 0.50 \mathrm{mmol}, 1.00 \mathrm{eq}$.) and 2,2-dimethylbutane (3 $\mathrm{mL}$ ) under reflux via syringe pump at a rate of $2 \mathrm{ml} / \mathrm{h}$. After addition was complete, the reaction mixture was stirred at $50{ }^{\circ} \mathrm{C}$ for $30 \mathrm{~min}$, and then allowed to cool to room temperature and concentrated in vacuo. ${ }^{1} \mathrm{H}$ NMR of the crude reaction mixture did not show any of the anti isomer, and so diastereoselectivity was assigned as $>95 \%$ de. The product was purified by flash chromatography on silica gel using 18:1 hexane/Et $\mathrm{E}_{2} \mathrm{O}$ as eluant to provide a white solid, $183 \mathrm{mg}(95 \%)$. Mp 66-68 ${ }^{\circ} \mathrm{C}$. [_]D +80.0 (ee 98\%) (c 1.54, $\left.\mathrm{CHCl}_{3}\right) .{ }^{1} \mathrm{H}$ NMR $\left(500 \mathrm{MHz}, \mathrm{CDCl}_{3}\right)$ : $7.44(\mathrm{~d}, 2 \mathrm{H}, J=7.0 \mathrm{~Hz}), 7.35-7.21(\mathrm{~m}, 8 \mathrm{H})$, $5.11(\mathrm{~d}, 1 \mathrm{H}, J=9.0 \mathrm{~Hz}), 3.80$ (d, 1H, J=9.0 Hz), 3.43 (s, 3H), 0.62 (s, 9H), -0.36 (s, 3H), 0.39 (s, 3H). ${ }^{13} \mathrm{C}$ NMR $\left(125 \mathrm{MHz}, \mathrm{CDCl}_{3}\right)$ :_172.4, 143.1, 136.7, 129.7, 128.4, 128.2, 127.9, 127.7, 127.2, 77.4, 61.9, 51.9, 25.7, 18.1, -4.7, -5.5. IR $\left(\mathrm{CHCl}_{3}\right): 2952,2929$, 
2856, 1735, 1250, 1160, $1090 \mathrm{~cm}^{-1} \cdot \mathrm{m} / z$ (ESI): $370\left(\mathrm{M}^{+}, 100 \%\right), 270$ (41\%), $239(46 \%)$.

Anal. calcd. for $\mathrm{C}_{22} \mathrm{H}_{30} \mathrm{O}_{3} \mathrm{Si}$ : C, 71.31: H, 8.16. Found: C, 71.32; H, 8.20. HPLC analysis: $98 \%$ ee (Chiralcel OD-H, $1 \% i-\mathrm{PrOH}$ in hexane, $1.0 \mathrm{ml} / \mathrm{min},=254 \mathrm{~nm}, t_{\mathrm{R}}=3.8 \mathrm{~min}$, minor; $t_{\mathrm{R}}=4.8 \mathrm{~min}$, major).

(2R,3R)-Methyl 3-(tert-butyldimethylsilyloxy)-3-(4-chlorophenyl)-2phenylpropanoate (8d)<smiles>COC(OC)[C@H](c1ccccc1)C(OC)c1ccc(Cl)cc1</smiles>

Procdure A: A solution of methyl phenyldiazoacetate 7 (352 mg, $2.00 \mathrm{mmol}, 2.00 \mathrm{eq}$.) in 2,2-dimethylbutane $(8 \mathrm{~mL})$ was added to a solution of $\mathrm{Rh}_{2}(R \text {-DOSP })_{4}(19 \mathrm{mg}, 0.01$ mmol, 0.01 eq.) and compound $\mathbf{6 d}(257 \mathrm{mg}, 1.00 \mathrm{mmol}, 1.00$ eq.) in 2,2-dimethylbutane $(1 \mathrm{~mL}$ ) via syringe pump at a rate of $4 \mathrm{ml} / \mathrm{h}$. After addition was complete, the reaction mixture was stirred for $1 \mathrm{~h}$, and then concentrated in vacuo. Diastereoselectivity ( $94 \%$ de) was determined by ${ }^{1} \mathrm{H}$ NMR of the crude reaction mixture, and the products purified by flash chromatography on silica gel using $18: 1$ hexane/ $\mathrm{Et}_{2} \mathrm{O}$ as eluant to isolate a mixture of anti and syn isomers as a colorless oil, $8 \mathrm{mg}$; and pure syn isomer as a white solid, 348 mg (total yield: $88 \%$ ).

Procdure B: A solution of methyl phenyldiazoacetate 7 (176 mg, $1.00 \mathrm{mmol}, 2.00$ eq.) in 2,2-dimethylbutane $(6 \mathrm{~mL})$ was added to a solution of $\mathrm{Rh}_{2}(S \text {-PTTL })_{4}(6 \mathrm{mg}, 0.005 \mathrm{mmol}$, 0.01 eq.) and compound $\mathbf{6 d}$ (129 mg, $0.50 \mathrm{mmol}, 1.00$ eq.) and 2,2-dimethylbutane (3 $\mathrm{mL}$ ) under reflux via syringe pump at a rate of $2 \mathrm{ml} / \mathrm{h}$. After addition was complete, the reaction mixture was stirred at $50{ }^{\circ} \mathrm{C}$ for $30 \mathrm{~min}$, and then allowed to cool to room temperature and concentrated in vacuo. ${ }^{1} \mathrm{H}$ NMR of the crude reaction mixture did not show any of the anti isomer, and so diastereoselectivity was assigned as $>95 \%$ de. The product was purified by flash chromatography on silica gel using 18:1 hexane/Et $\mathrm{t}_{2} \mathrm{O}$ as eluant to provide a white solid, $171 \mathrm{mg}(84 \%)$. Mp $47-49{ }^{\circ} \mathrm{C}$. [_]D +87.8 (ee 97\%) (c 1.54, $\left.\mathrm{CHCl}_{3}\right) .{ }^{1} \mathrm{H} \mathrm{NMR}\left(500 \mathrm{MHz}, \mathrm{CDCl}_{3}\right)$ : $7.39(\mathrm{~d}, 2 \mathrm{H}, J=7.0 \mathrm{~Hz}), 7.38-7.24(\mathrm{~m}, 7 \mathrm{H})$, 5.09 (d, 1H, $J=9.0 \mathrm{~Hz}), 3.74(\mathrm{~d}, 1 \mathrm{H}, J=9.0 \mathrm{~Hz}), 3.45$ (s, 3H), $0.62(\mathrm{~s}, 9 \mathrm{H}),-0.38(\mathrm{~s}, 3 \mathrm{H}),-$ 0.39 (s, 3H). ${ }^{13} \mathrm{C}$ NMR $\left(125 \mathrm{MHz}, \mathrm{CDCl}_{3}\right)$ :_172.2, 141.7, 136.3, 133.6, 129.7, 128.6, 
128.4 (2C), 127.8, 76.7, 61.7, 52.0, 25.8, 18.1, -4.7, -5.5. IR $\left(\mathrm{CHCl}_{3}\right): 2952,2929,2856$, 2886, 1736, 1487, $1092 \mathrm{~cm}^{-1} . \mathrm{m} / z$ (ESI): $405\left(\mathrm{M}^{+}, 30 \%\right), 391$ (100\%), 299 (21\%), 277 (29\%), 258 (49\%). Anal. calcd. for $\mathrm{C}_{22} \mathrm{H}_{29} \mathrm{ClO}_{3} \mathrm{Si}$ : C, 65.24: H, 7.22. Found: C, 65.29; H, 7.27. HPLC analysis: $97 \%$ ee (Chiralcel OD-H, $1 \% i-\mathrm{PrOH}$ in hexane, $1.0 \mathrm{ml} / \mathrm{min},=254$ $\mathrm{nm}, t_{\mathrm{R}}=3.8 \mathrm{~min}$, minor; $t_{\mathrm{R}}=4.6$ min, major).

\section{(2R,3R)-Methyl 3-(tert-butyldimethylsilyloxy)-2-phenyl-3-(4-}

(trifluoromethyl)phenyl)propanoate (8e)

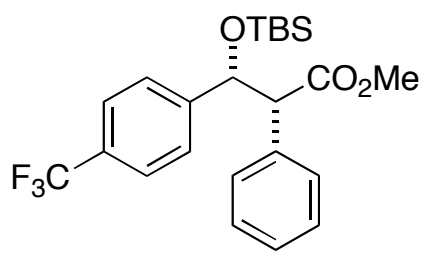

Procdure A: A solution of methyl phenyldiazoacetate 7 (352 mg, $2.00 \mathrm{mmol}, 2.00$ eq.) in 2,2-dimethylbutane ( $8 \mathrm{~mL})$ was added to a solution of $\mathrm{Rh}_{2}(R \text {-DOSP })_{4}(19 \mathrm{mg}, 0.01$ mmol, 0.01 eq.) and compound $6 \mathbf{e}(290 \mathrm{mg}, 1.00 \mathrm{mmol}, 1.00$ eq.) in 2,2-dimethylbutane $(1 \mathrm{~mL}$ ) via syringe pump at a rate of $4 \mathrm{ml} / \mathrm{h}$. After addition was complete, the reaction mixture was stirred for $1 \mathrm{~h}$, and then concentrated in vacuo. Diastereoselectivity ( $93 \% \mathrm{de}$ ) was determined by ${ }^{1} \mathrm{H}$ NMR of the crude reaction mixture, and the products purified by flash chromatography on silica gel using $18: 1$ hexane/ $\mathrm{Et}_{2} \mathrm{O}$ as eluant to isolate a mixture of the anti and syn isomers as a white solid, $28 \mathrm{mg}$; and pure syn isomer as a white solid, $296 \mathrm{mg}$ (total yield: 74\%).

Procdure B: A solution of methyl phenyldiazoacetate 7 (176 mg, $1.00 \mathrm{mmol}, 2.00$ eq.) in 2,2-dimethylbutane $(6 \mathrm{~mL})$ was added to a solution of $\mathrm{Rh}_{2}(S \text {-PTTL })_{4}(6 \mathrm{mg}, 0.005 \mathrm{mmol}$, 0.01 eq.) and compound $6 \mathbf{e}(145 \mathrm{mg}, 0.50 \mathrm{mmol}, 1.00 \mathrm{eq}$.) and 2,2-dimethylbutane (3 $\mathrm{mL}$ ) under reflux via syringe pump at a rate of $2 \mathrm{ml} / \mathrm{h}$. After addition was complete, the reaction mixture was stirred at $50{ }^{\circ} \mathrm{C}$ for $30 \mathrm{~min}$, and then allowed to cool to room temperature and concentrated in vacuo. ${ }^{1} \mathrm{H}$ NMR of the crude reaction mixture did not show any of the anti isomer, and so diastereoselectivity was assigned as $>95 \% \mathrm{de}$. The product was purified by flash chromatography on silica gel using 18:1 hexane/Et $\mathrm{E}_{2} \mathrm{O}$ as eluant to provide a white solid, $197 \mathrm{mg}(90 \%)$. Mp 70-73 ${ }^{\circ} \mathrm{C}$. [_]D $+68.0($ ee $95 \%)(c$ 1.13, $\left.\mathrm{CHCl}_{3}\right) .{ }^{1} \mathrm{H}$ NMR $\left(500 \mathrm{MHz}, \mathrm{CDCl}_{3}\right):=7.54(\mathrm{~d}, 2 \mathrm{H}, J=8.0 \mathrm{~Hz}), 7.46(\mathrm{~d}, 2 \mathrm{H}, J=8.0$ $\mathrm{Hz}), 7.39$ (d, 2H, J=7.0 Hz), 7.33-7.25 (m, 3H), 5.18 (d, 1H, J=8.5 Hz), 3.77 (d, 1H, 
$J=8.5 \mathrm{~Hz}), 3.45$ (s, 3H), 0.64 (s, 9H), -0.37 (s, 3H), -0.39 (s, 3H). ${ }^{13} \mathrm{C}$ NMR $(125 \mathrm{MHz}$, $\left.\mathrm{CDCl}_{3}\right)$ :_171.8, 146.9, 135.8, 129.8 (q, $\left.J=32.0 \mathrm{~Hz}\right), 129.4,128.2,127.6,127.3,124.9$ (q, $J=4.0 \mathrm{~Hz}), 124.1$ (q, $J=272.0 \mathrm{~Hz}), 76.4,61.3,51.7,25.4,17.8,-5.0,-5.8$. IR $\left(\mathrm{CHCl}_{3}\right)$ : 2954, 2930, 2858, 1736, 1326, $1127 \mathrm{~cm}^{-1} . \mathrm{m} / z$ (ESI): $439\left(\mathrm{MH}^{+}, 55 \%\right), 391$ (41\%), 236 (100\%). Anal. calcd. for $\mathrm{C}_{23} \mathrm{H}_{29} \mathrm{~F}_{3} \mathrm{O}_{3} \mathrm{Si}$ : C, 62.99: H, 6.67. Found: C, 63.13; H, 6.17. HPLC analysis: 30\%ee (Chiralcel OD-H, 1\% $i-\mathrm{PrOH}$ in hexane, $1.0 \mathrm{ml} / \mathrm{min},=254 \mathrm{~nm}$, $t_{\mathrm{R}}=3.6 \mathrm{~min}$, minor; $t_{\mathrm{R}}=4.4 \mathrm{~min}$, major.

(2R,3R)-Methyl 3-(4-methoxyphenyl)-2-phenyl-3-(trimethylsilyloxy)propanoate (10a)

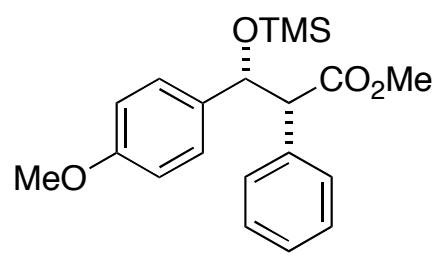

A solution of methyl phenyldiazoacetate 7 (352 $\mathrm{mg}, 2.00 \mathrm{mmol}, 2.00$ eq.) in 2,2dimethylbutane $(8 \mathrm{~mL})$ was added to a solution of $\mathrm{Rh}_{2}(R \text {-DOSP })_{4}(19 \mathrm{mg}, 0.01 \mathrm{mmol}$, 0.01 eq.) and (4-methoxybenzyloxy)trimethylsilane (210 mg, $1.00 \mathrm{mmol}, 1.00$ eq.) in 2,2dimethylbutane $(1 \mathrm{~mL})$ via syringe pump at a rate of $4 \mathrm{ml} / \mathrm{h}$. After addition was complete, the reaction mixture was stirred for $1 \mathrm{~h}$, and then concentrated in vacuo. Diastereoselectivity $\left(83 \%\right.$ de) was determined by ${ }^{1} \mathrm{H}$ NMR of the crude reaction mixture, and the products purified by flash chromatography on silica gel using $18: 1$ hexane/Et ${ }_{2} \mathrm{O}$ as eluant to isolate a mixture of anti and syn products as a colorless oil, $54 \mathrm{mg}$; and pure syn isomer as a white solid, $199 \mathrm{mg}$ (total yield: $71 \%$ ). $\mathrm{Mp} 81-83{ }^{\circ} \mathrm{C}$. [ ] $]_{\mathrm{D}}+29.7$ (ee 38\%) (c 1.54, $\left.\mathrm{CHCl}_{3}\right)$. ${ }^{1} \mathrm{H}$ NMR $\left(\mathrm{CDCl}_{3}\right)$ : 7.41 (d, 2H, J=7.5 Hz), 7.32-7.25 (m, 5H), 6.81 (d, 2H, $J=8.0 \mathrm{~Hz}$ ), 5.07 (d, 1H, J=9.0 Hz), 3.79 (s, 3H), 3.79 (d, 1H, J=9.0 Hz), 3.44 (s, 3H), -0.27 (s, 9H). ${ }^{13} \mathrm{C}$ NMR $\left(\mathrm{CDCl}_{3}\right): \_172.2,158.9,136.6,134.9,129.1,128.0,127.9,127.2$, 113.3, 76.3, 61.3, 55.1, 51.6, -0.4. IR $\left(\mathrm{CHCl}_{3}\right): 3001,2954,2838,1735,1611,1511$, 1455, 1250, $1081 \mathrm{~cm}^{-1} . \mathrm{m} / z$ (ESI): $381\left(\mathrm{MNa}^{+}, 6 \%\right), 285$ (1\%), 269 (100\%). Anal. calcd. for $\mathrm{C}_{20} \mathrm{H}_{26} \mathrm{O}_{4} \mathrm{Si}$ : C, 67.00: H, 7.31. Found: C, 67.03; H, 7.29. HPLC analysis: 38\%ee (Chiralcel OD-H, $1 \% i$-PrOH in hexane, $1.0 \mathrm{ml} / \mathrm{min},{ }_{-}=254 \mathrm{~nm}, t_{\mathrm{R}}=5.2 \mathrm{~min}$, minor; $t_{\mathrm{R}}=6.1$ min, major). 

(10b)

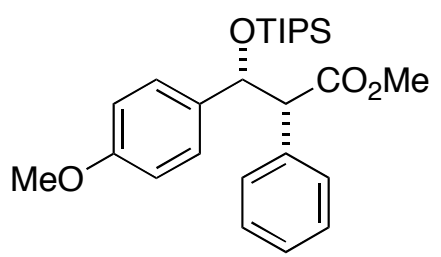

A solution of methyl phenyldiazoacetate 7 (264 mg, $1.50 \mathrm{mmol}, 2.0$ eq.) in 2,2dimethylbutane $(5 \mathrm{~mL})$ was added to a solution of $\mathrm{Rh}_{2}(R \text {-DOSP })_{4}(14 \mathrm{mg}, 0.075 \mathrm{mmol}$, 0.01 eq.) and (4-methoxybenzyloxy)triisopropylsilane (221 mg, $0.75 \mathrm{mmol}, 1$ eq.) and 2,2-dimethylbutane $(1 \mathrm{~mL})$ in 2,2-dimethylbutane $(1 \mathrm{~mL})$ via syringe pump at a rate of $2.5 \mathrm{ml} / \mathrm{h}$. After addition was complete, the reaction mixture was stirred for $1 \mathrm{~h}$, and then concentrated in vacuo. ${ }^{1} \mathrm{H}$ NMR of the crude reaction mixture did not show any of the anti isomer, so diastereoselectivity was assigned as $>95 \%$. The product was purified by flash chromatography on silica using 18:1 hexane/ $\mathrm{Et}_{2} \mathrm{O}$ to isolate a colorless oil, $152 \mathrm{mg}$ (46\%). [ ] $]_{\mathrm{D}}-11.18$ (ee 15\%) (c 1.52, $\left.\mathrm{CHCl}_{3}\right) .{ }^{1} \mathrm{H}$ NMR $\left(\mathrm{CDCl}_{3}\right)$ : 7.44 (d, 2H, J=7.0 Hz), 7.31-7.25 (m, 5H), $6.81(\mathrm{~d}, 2 \mathrm{H}, J=8.5 \mathrm{~Hz}), 5.23$ (d, 1H, J=9.0 Hz), $3.83(\mathrm{~d}, 1 \mathrm{H}$, $J=9.0 \mathrm{~Hz}), 3.79(\mathrm{~s}, 3 \mathrm{H}), 3.41(\mathrm{~s}, 3 \mathrm{H}), 0.78-0.71(\mathrm{~m}, 21 \mathrm{H}) .{ }^{13} \mathrm{C} \mathrm{NMR}\left(\mathrm{CDCl}_{3}\right):{ }_{-} 172.1$, $159.0,136.4$, 135.1, 129.5, 128.4, 128.1, 127.4, 113.1, 77.1, 62.1, 55.1, 51.5, 17.7, 12.3 . IR $\left(\mathrm{CHCl}_{3}\right): 2956,2838,1733,1512,1455,1253,1081 \mathrm{~cm}^{-1} . m / z(\mathrm{EI}): 442\left(\mathrm{M}^{+}, 9 \%\right), 411$ (6\%), 399 (8\%), 305 (100\%), 293 (46\%), 269 (7\%), 145 (46\%), 121 (49\%), 89 (88\%). Anal. calcd. for $\mathrm{C}_{26} \mathrm{H}_{38} \mathrm{O}_{4} \mathrm{Si}$ : C, 70.55: H, 8.65. Found: C, 70.48; H, 8.70. HPLC analysis: $15 \%$ ee (Chiralcel OD-H, $1 \% i-\mathrm{PrOH}$ in hexane, $1.0 \mathrm{ml} / \mathrm{min},{ }_{-}=254 \mathrm{~nm}, t_{\mathrm{R}}=5.5 \mathrm{~min}$, minor; $t_{\mathrm{R}}=7.1 \mathrm{~min}$, major).

(2S,3S)-tert-Butyl 3-(tert-butyldimethylsilyloxy)-3-(4-methoxyphenyl)-2phenylpropanoate (10c)<smiles>COc1ccc([C@H](O[AsH3])[C@H](C(=O)OC(C)(C)C)c2ccccc2)cc1</smiles>

A solution of tert-butyl phenyldiazoacetate 9 (436 mg, $2.00 \mathrm{mmol}, 2.00$ eq.) in 2,2dimethylbutane $(8 \mathrm{~mL})$ was added to a solution of $\mathrm{Rh}_{2}(R \text {-DOSP })_{4}(19 \mathrm{mg}, 0.01 \mathrm{mmol}$, 
0.01 eq.) and compound $6 \mathbf{6}(252 \mathrm{mg}, 1.00 \mathrm{mmol}, 1.00$ eq.) in 2,2-dimethylbutane (1 mL) via syringe pump at a rate of $4 \mathrm{ml} / \mathrm{h}$. After addition was complete, the reaction mixture was stirred for $1 \mathrm{~h}$, and then concentrated in vacuo. Diastereoselectivity ( $78 \%$ de) was determined by ${ }^{1} \mathrm{H}$ NMR of the crude reaction mixture, and the products purified by flash chromatography on silica gel using 18:1 hexane/ $\mathrm{Et}_{2} \mathrm{O}$ as eluant to isolate the anti isomer as a white solid, $31 \mathrm{mg}$; a mixture of the anti and syn isomers as a white solid, $110 \mathrm{mg}$; and the pure syn isomer as a white solid, $209 \mathrm{mg}$ (total yield: 73\%). syn isomer: Mp 79$81{ }^{\circ} \mathrm{C}$. [ $]_{\mathrm{D}}-30.2$ (ee 74\%) (c 1.62, $\left.\mathrm{CHCl}_{3}\right) .{ }^{1} \mathrm{H}$ NMR $\left(500 \mathrm{MHz}, \mathrm{CDCl}_{3}\right)$ : _7.46 (d, 2H, $J=7.5 \mathrm{~Hz}$ ), 7.31-7.26 (m, 5H), 6.82 (d, 2H, $J=8.0 \mathrm{~Hz}), 4.92$ (d, 1H, $J=9.0 \mathrm{~Hz}), 3.79$ (s, $3 \mathrm{H}), 3.68(\mathrm{~d}, 1 \mathrm{H}, J=9.0 \mathrm{~Hz}), 1.14$ (s, 9H), 0.57 (s, 9H), -0.43 (s, 3H), $-0.44(\mathrm{~s}, 3 \mathrm{H}) .{ }^{13} \mathrm{C}$ NMR (125 MHz, $\left.\mathrm{CDCl}_{3}\right)$ : $137.7,135.4,130.1,129.4,129.1,128.8,128.3,127.3,80.8$, 77.5, 62.8, 55.4, 28.3, 27.9, 25.7, 18.0, -4.6, -5.6. IR $\left(\mathrm{CHCl}_{3}\right): 2955,2930,2856,1724$, 1512, 1368, 1252, 1148, $1081 \mathrm{~cm}^{-1} . \mathrm{m} / z$ (ESI): $465\left(\mathrm{MNa}^{+}, 100 \%\right), 462(67 \%), 349$ (15\%), 310 (39\%). Anal. calcd. for $\mathrm{C}_{26} \mathrm{H}_{38} \mathrm{O}_{4} \mathrm{Si}$ : C, 70.55: H, 8.65. Found: C, 70.58; H, 8.69. HPLC analysis determined by conversion of the product 10 $\mathrm{c}$ to the alcohol 13a: $74 \%$ ee $\left(R, R\right.$-Whelk-O1, $3 \% i$-PrOH in hexane, $1.0 \mathrm{ml} / \mathrm{min},{ }_{-}=254 \mathrm{~nm}, t_{\mathrm{R}}=10.1 \mathrm{~min}$, major; $t_{\mathrm{R}}=11.9$ min, minor.

\section{(S)-Ethyl 2-(2-phenylacetoxy)propanoate}<smiles>CCOC(=O)C(C)OC(=O)Cc1ccccc1</smiles>

1,3-Dicyclohexylcarbodiimide (1.0 $\mathrm{M}$ in $\mathrm{CH}_{2} \mathrm{Cl}_{2}, 30 \mathrm{~mL}, 30 \mathrm{mmol}, 1$ eq.) was added dropwise via syringe to a cold $\left(0{ }^{\circ} \mathrm{C}\right)$ solution of phenylacetic acid $(4.62 \mathrm{~g}, 30 \mathrm{mmol}, 1$ eq.), $N, N$-dimethylaminopyridine ( $366 \mathrm{mg}, 3 \mathrm{mmol}, 0.1$ eq.) and ethyl (S)-lactate (6.81 $\mathrm{mL}, 20 \mathrm{mmol}, 2$ eq.) in $\mathrm{CH}_{2} \mathrm{Cl}_{2}(150 \mathrm{~mL})$. A white precipitate began to form, and the reaction mixture was allowed to reach room temperature over $12 \mathrm{~h}$. The suspension was then filtered through Celite ${ }^{\circledR}$, and washed with $\mathrm{CH}_{2} \mathrm{Cl}_{2}$. The filtrate was concentrated in vacuo, and purified by chromatography on silica gel using 10:1 hexane/ $\mathrm{Et}_{2} \mathrm{O}$ as eluant to isolate a colorless oil, $6.55 \mathrm{~g}(92 \%)$. [ $]_{\mathrm{D}}-34.95$ (c 1.83, $\left.\mathrm{CHCl}_{3}\right) .{ }^{1} \mathrm{H}$ NMR $(400 \mathrm{MHz}$, $\mathrm{CDCl}_{3}$ ):_7.39-7.25 (m, 5H), 5.09 (q, $\left.1 \mathrm{H}, J=7.0 \mathrm{~Hz}\right), 4.18$ (q, $2 \mathrm{H}, J=7.0 \mathrm{~Hz}$ ), 3.74-3-69 $(\mathrm{m}, 2 \mathrm{H}), 1.50(\mathrm{~d}, 3 \mathrm{H}, J=7.0 \mathrm{~Hz}), 1.24(\mathrm{t}, 3 \mathrm{H}, J=7.0 \mathrm{~Hz}) .{ }^{13} \mathrm{C} \mathrm{NMR}\left(75 \mathrm{MHz}, \mathrm{CDCl}_{3}\right)$ : 
170.9, 170.6, 133.5, 129.3, 128.5, 127.1, 69.0, 61.3, 40.8, 16.8, 14.0. IR (film): 2988, 1742, 1604, 1456, 1354, 1210, $1098 \mathrm{~cm}^{-1} . \mathrm{m} / \mathrm{z}$ (EI): $236\left(77 \%, \mathrm{M}^{+}\right), 145(60 \%), 118$ (100\%), 105 (41\%), 91 (98\%). Anal. calcd. for $\mathrm{C}_{13} \mathrm{H}_{16} \mathrm{O}_{4}: \mathrm{C}, 66.09$; H, 6.83. Found: $\mathrm{C}$, $66.46 ; \mathrm{H}, 6.80$.

\section{(S)-2-(2-Diazo-2-phenyl-acetoxy)-propionic acid ethyl ester (11)}<smiles>CCOC(=O)C(C)OC(=O)C(=N)c1ccccc1</smiles>

A solution of (S)-ethyl 2-(2-phenylacetoxy)propanoate $(6.00 \mathrm{~g}, 25.39 \mathrm{mmol}, 1.0$ eq.) and para-acetaminobenzenesulfonyl azide $(6.39 \mathrm{~g}, 27.93 \mathrm{mmol}, 1.1 \mathrm{eq}$.) in acetonitrile ( 25 $\mathrm{mL}$ ) was cooled to $0{ }^{\circ} \mathrm{C}$, and 1,8-diazabicyclo[5.4.0]undec-7-ene (3.61 mL, $24.12 \mathrm{mmol}$, 0.9 eq.) was added dropwise via syringe over $15 \mathrm{~min}$. The reaction mixture was allowed to reach room temperature over $12 \mathrm{~h}$. The reaction was quenched with saturated $\mathrm{NH}_{4} \mathrm{Cl}$ solution, and then extracted with $\mathrm{CH}_{2} \mathrm{Cl}_{2}(3 \mathrm{x})$. The combined organic extracts were washed with brine, dried over $\mathrm{Na}_{2} \mathrm{SO}_{4}$, and concentrated in vacuo. The residue was triturated with 1:1 hexane/Et $2 \mathrm{O}$, filtered through Celite ${ }^{\circledR}$, and concentrated in vacuo. The residue was purified by chromatography on silica gel using 10:1 hexane/Et $\mathrm{t}_{2} \mathrm{O}$ as eluant to isolate an orange oil, $5.92 \mathrm{~g}(89 \%)$. [_] $\mathrm{D}+5.9\left(c \quad 1.80, \mathrm{CHCl}_{3}\right) .{ }^{1} \mathrm{H}$ NMR $(400 \mathrm{MHz}$, $\mathrm{CDCl}_{3}$ ):_7.49 (d, 2H, J=7.5 Hz), 7.39 (t, 2H, $\left.J=8.0 \mathrm{~Hz}\right), 7.20$ (t, 1H, $\left.J=7.0 \mathrm{~Hz}\right), 5.27$ (q, $1 \mathrm{H}, J=7.0 \mathrm{~Hz}$ ), 4.24 (q, 2H, $J=7.0 \mathrm{~Hz}$ ), 1.57 (d, 3H, $J=7.0 \mathrm{~Hz}$ ), 1.31 (t, $3 \mathrm{H}, J=7.0 \mathrm{~Hz}$ ). ${ }^{13} \mathrm{C}$ NMR (125 MHz, $\left.\mathrm{CDCl}_{3}\right)$ :_170.5, 164.3, 128.8, 125.8, 125.0, 123.8, 68.8, 61.3, 16.9, 13.9. The carbon attached to the diazo moiety was not observed. IR $\left(\mathrm{CH}_{2} \mathrm{Cl}_{2}\right): 2988$, 2090, 1755, 1708, 1599, 1499, 1451, 1155, $1098 \mathrm{~cm}^{-1} . \mathrm{m} / z$ (EI): 262 ( $\left.\mathrm{M}^{+}, 9 \%\right), 189$ (8\%), 117 (9\%), 105 (100\%), 89 (34\%), 77 (32\%), 63 (16\%). HRMS Calcd. for $\mathrm{C}_{13} \mathrm{H}_{14} \mathrm{O}_{4} \mathrm{~N}_{2}$ : 262.0948. Found: 262.0957. 
(2R, 3R)-3-(tert-Butyl-dimethyl-silanyloxy)-3-(4-methoxy-phenyl)-2-phenyl-

propionic acid (1S)-1-ethoxycarbonyl-ethyl ester (12a)

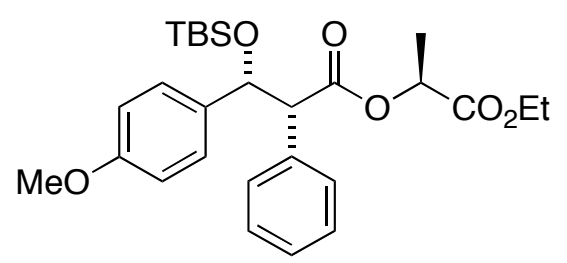

A solution of $\mathrm{Rh}_{2}(\mathrm{OOct})_{4}(3.9 \mathrm{mg}, 0.005 \mathrm{mmol}, 0.01$ eq.), compound 6a (126 mg, 0.5 mmol, 1 eq.) and 2,2-dimethylbutane $(5 \mathrm{~mL})$ was heated under reflux for $45 \mathrm{~min}$ to dissolve the catalyst, and then allowed to cool to room temperature. A solution of compound 11 (262 mg, $1.0 \mathrm{mmol}, 2$ eq.) in 2,2-dimethylbutane (10 mL) was added via syringe pump at a rate of $3.33 \mathrm{ml} / \mathrm{h}$. After addition was complete, the reaction mixture was stirred for $1 \mathrm{~h}$, and then concentrated in vacuo. syn/anti ratio (12.9:1) was determined by ${ }^{1} \mathrm{H}$ NMR of the crude reaction mixture, and the product purified by flash chromatography on silica gel using 10:1 hexane/ $\mathrm{Et}_{2} \mathrm{O}$ as eluant to isolate an inseparable mixture of the all anti and syn isomers as a colorless oil, $201 \mathrm{mg}$ (83\%). Major syn isomer: ${ }^{1} \mathrm{H}$ NMR (500 MHz, $\left.\mathrm{CDCl}_{3}\right)$ : $7.45(\mathrm{~d}, 2 \mathrm{H}, J=7.5 \mathrm{~Hz}), 7.33-7.24(\mathrm{~m}, 5 \mathrm{H}), 6.82$ $(\mathrm{d}, 2 \mathrm{H}, J=8.5 \mathrm{~Hz}), 5.04$ (d, 1H, $J=8.0 \mathrm{~Hz}), 4.81(1 \mathrm{H}, \mathrm{q}, J=7.0 \mathrm{~Hz}), 4.06-3.98(\mathrm{~m}, 2 \mathrm{H})$, $3.87(\mathrm{~d}, 1 \mathrm{H}, J=9.0 \mathrm{~Hz}), 3.81(\mathrm{~s}, 3 \mathrm{H}), 1.16(\mathrm{~d}, 3 \mathrm{H}, J=7.0 \mathrm{~Hz}), 1.07$ (t, 3H, $J=7.0 \mathrm{~Hz}), 0.62$ $(\mathrm{s}, 9 \mathrm{H}),-0.38(\mathrm{~s}, 3 \mathrm{H}),-0.39(\mathrm{~s}, 3 \mathrm{H})$. The tert-butyl singlet for other diastereoisomers come into resonance at 0.84 (anti), _0.83 (anti), 0.60 (syn) $){ }^{13} \mathrm{C}$ NMR $\left(\mathrm{CDCl}_{3}, 125\right.$ MHz):_170.9, 170.1, 159.0, 136.0, 134.7, 129.4, 128.2, 127.9, 127.2, 113.1, 76.8, 68.5, $61.1,61.0,55.0,25.4,17.7,16.5,13.7,-5.1,-5.9$. IR $\left(\mathrm{CH}_{2} \mathrm{Cl}_{2}\right): 2932,2860,1740,1693$, 1513, $1082 \mathrm{~cm}^{-1} \cdot \mathrm{m} / z(\mathrm{EI}): 509\left(\mathrm{MNa}^{+}, 100 \%\right), 269$ (25\%), 237 (20\%), 209 (15\%). Anal. calcd. for $\mathrm{C}_{27} \mathrm{H}_{38} \mathrm{O}_{6}$ Si: C, 66.63; H, 7.87. Found: C, 66.36; H, 7.85. 


\section{(2S,3R)-3-(tert-Butyldimethylsilyloxy)-3-(4-methoxyphenyl)-2-phenylpropan-1-ol}

(13a)

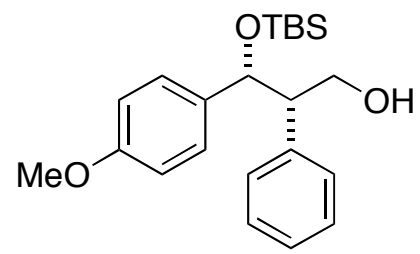

A solution of $\mathrm{Rh}_{2}(\mathrm{OOct})_{4}(3.9 \mathrm{mg}, 0.005 \mathrm{mmol}, 0.01 \mathrm{eq}$ ), compound $6 \mathbf{a}(126 \mathrm{mg}, 0.5$ mmol, 1 eq.) and 2,2-dimethylbutane (5 mL) was heated under reflux for $45 \mathrm{~min}$, then allowed to cool to room temperature. A solution of compound 11 (262 mg, $1.0 \mathrm{mmol}, 2$ eq.) in 2,2-dimethylbutane $(10 \mathrm{~mL})$ was added via syringe pump at a rate of $3.33 \mathrm{ml} / \mathrm{h}$. After addition was complete, the reaction mixture was stirred for $1 \mathrm{~h}$, and then cooled to $0{ }^{\circ} \mathrm{C}$. Diisobutylaluminium hydride $(1.0 \mathrm{M}$ in toluene, $3.0 \mathrm{ml}, 3.0 \mathrm{mmol}, 6 \mathrm{eq}$.) was added, and the reaction stirred for $2 \mathrm{~h}$ at $0{ }^{\circ} \mathrm{C}$ and then $10 \mathrm{~h}$ at room temperature. The reaction mixture was then poured into a 1:1 mix $(50 \mathrm{~mL})$ of $\mathrm{Et}_{2} \mathrm{O}$ and saturated sodium potassium tartrate solution. The biphasic mixture was stirred for $2 \mathrm{~h}$. The layers were separated, and the aqueous layer was extracted with 2 portions $\mathrm{Et}_{2} \mathrm{O}$. The combined organic fractions were dried $\left(\mathrm{Na}_{2} \mathrm{SO}_{4}\right)$ and concentrated in vacuo. Diastereoselectivity ( $83 \%$ de) was determined by ${ }^{1} \mathrm{H}$ NMR of the crude reaction mixture, and the product purified by flash chromatography on silica gel using 10:1 hexane/ $\mathrm{Et}_{2} \mathrm{O}$ as eluant to isolate a mixture of the anti and syn isomers as a colorless oil, $53 \mathrm{mg}$; and the pure syn isomer as a colorless oil, $78 \mathrm{mg}$ (total yield: 70\%). [_] $\mathrm{D}+38.2$ (ee 79\%) (c 1.22, $\left.\mathrm{CHCl}_{3}\right) .{ }^{1} \mathrm{H}$ NMR $(500 \mathrm{MHz}$, $\left.\mathrm{CDCl}_{3}\right)$ :_7.26-7.21 (m, 3H), 7.08-7.05 (m, 2H), $6.99(\mathrm{~d}, 2 \mathrm{H}, J=8.5 \mathrm{~Hz}), 6.77(\mathrm{~d}, 2 \mathrm{H}$, $J=8.5 \mathrm{~Hz}$ ), 4.91 (d, 1H, $J=7.0 \mathrm{~Hz}$ ), 3.88 (dd, 1H, $J=11.0,7.5 \mathrm{~Hz}$ ), 3.80 (s, 3H), 3.77 (dd, $1 \mathrm{H}, J=11.0,7.0 \mathrm{~Hz}$ ), 3.15 (dd, 1H, $J=13.0,7.0 \mathrm{~Hz}$ ), 1.21 (br s, 1H), 0.79 (s, 9H), -0.10 (s, $3 \mathrm{H}),-0.27$ (s, 3H). ${ }^{13} \mathrm{C}$ NMR (125 MHz, $\left.\mathrm{CDCl}_{3}\right)$ : $158.8,139.1,134.4,129.3,128.0$, $127.9,126.8,113.1,77.0,63.7,56.4,55.1,25.7,18.0,-4.8,-5.5$. IR $\left(\mathrm{CHCl}_{3}\right): 3456,2958$, 1512, 1248, $1083 \mathrm{~cm}^{-1} \cdot \mathrm{m} / z(\mathrm{EI}): 373\left(\mathrm{M}^{+}, 1 \%\right), 357$ (36\%), 251 (100\%). Anal. Calcd. for $\mathrm{C}_{22} \mathrm{H}_{32} \mathrm{O}_{3} \mathrm{Si}: \mathrm{C}, 70.92 ; \mathrm{H}, 8.66$. Found: $\mathrm{C}, 70.81 ; \mathrm{H}, 8.62$. HPLC analysis: $79 \%$ ee $(R, R-$ Whelk-O1, $3 \% i$-PrOH in hexane, $1.0 \mathrm{ml} / \mathrm{min},=254 \mathrm{~nm}, t_{\mathrm{R}}=10.1 \mathrm{~min}$, minor; $t_{\mathrm{R}}=11.9$ min, major). 


\section{(2S,3R)-3-(tert-Butyldimethylsilyloxy)-2-phenyl-3-p-tolylpropan-1-ol (13b)}<smiles>Cc1ccc([C@H]([O-])[C@H](CO)c2ccccc2)cc1</smiles>

A solution of $\mathrm{Rh}_{2}(\mathrm{OOct})_{4}(3.9 \mathrm{mg}, 0.005 \mathrm{mmol}, 0.01$ eq.), compound $\mathbf{6 b}$ (118 mg, 0.5 mmol, 1 eq.) and 2,2-dimethylbutane (5 mL) was heated under reflux for $30 \mathrm{~min}$ and then allowed to cool to room temperature. A solution of compound 11 (262 mg, $1.0 \mathrm{mmol}, 2$ eq.) in 2,2-dimethylbutane $(10 \mathrm{~mL})$ was added via syringe pump at a rate of $3.33 \mathrm{ml} / \mathrm{h}$. After addition was complete, the reaction mixture was stirred for $1 \mathrm{~h}$, and then cooled to $0{ }^{\circ} \mathrm{C}$. Diisobutylaluminium hydride $(1.0 \mathrm{M}$ in toluene, $3.0 \mathrm{ml}, 3.0 \mathrm{mmol}, 6$ eq. $)$ was added, and the reaction stirred for $2 \mathrm{~h}$ at $0{ }^{\circ} \mathrm{C}$ and then $10 \mathrm{~h}$ at room temperature. The reaction mixture was then poured into a 1:1 mix $(50 \mathrm{~mL})$ of $\mathrm{Et}_{2} \mathrm{O}$ and saturated sodium potassium tartrate solution. The biphasic mixture was stirred for $2 \mathrm{~h}$. The layers were separated, and the aqueous layer was extracted with 2 portions $\mathrm{Et}_{2} \mathrm{O}$. The combined organic fractions were dried $\left(\mathrm{Na}_{2} \mathrm{SO}_{4}\right)$ and concentrated in vacuo. Diastereoselectivity (79\% de) was determined by ${ }^{1} \mathrm{H}$ NMR of the crude reaction mixture, and the product purified by flash chromatography on silica gel using 10:1 hexane/ $\mathrm{Et}_{2} \mathrm{O}$ as eluant to isolate a mixture of the anti and syn isomers as a colorless oil, $23 \mathrm{mg}$; and the pure syn isomer as a colorless oil, $130 \mathrm{mg}$ (total yield: 86\%). [ [ ] $\mathrm{D}$ +48.0 (ee 80\%) (c 1.02, $\left.\mathrm{CHCl}_{3}\right) .{ }^{1} \mathrm{H}$ NMR $(500 \mathrm{MHz}$, $\left.\mathrm{CDCl}_{3}\right):{ }_{-} 7.28-7.20(\mathrm{~m}, 3 \mathrm{H}), 7.10-7.06(\mathrm{~m}, 2 \mathrm{H}), 7.04(\mathrm{~d}, 2 \mathrm{H}, J=7.5 \mathrm{~Hz}), 6.97(\mathrm{~d}, 2 \mathrm{H}$, $J=8.0 \mathrm{~Hz}), 4.91(\mathrm{~d}, 1 \mathrm{H}, J=6.0 \mathrm{~Hz}), 3.86(\mathrm{dd}, 1 \mathrm{H}, J=11.0,7.5 \mathrm{~Hz}), 3.76(\mathrm{dd}, 1 \mathrm{H}, J=11.0$, $7.0 \mathrm{~Hz}$ ), 3.14 (dd, 1H, J=7.0, $6.0 \mathrm{~Hz}), 2.32$ (s, 3H), 1.81 (br s, 1H), 0.79 (s, 9H), -0.11 (s, $3 \mathrm{H}),-0.27(\mathrm{~s}, 3 \mathrm{H}) .{ }^{13} \mathrm{C} \mathrm{NMR}\left(125 \mathrm{MHz}, \mathrm{CDCl}_{3}\right)$ : 139.2, 136.8, 129.3 (2C), 128.4, 128.0, 126.7, 126.6, 77.2, 63.7, 56.4, 25.7, 21.1, 18.0, -4.8, -5.5. IR (film): 3367, 2954, 1604, 1514, 1456, 1256, $1081 \mathrm{~cm}^{-1} . \mathrm{m} / z$ (ESI): $379\left(\mathrm{MNa}^{+}, 100 \%\right), 255(10 \%)$. Anal Calcd. for $\mathrm{C}_{22} \mathrm{H}_{32} \mathrm{O}_{2} \mathrm{Si}: \mathrm{C}, 74.10 ; \mathrm{H}, 9.05$. Found: $\mathrm{C}, 73.81 ; \mathrm{H}, 9.16$. HPLC analysis: $80 \%$ ee $(R, R-$ Whelk-O1, 3\% $i-\mathrm{PrOH}$ in hexane, $1.0 \mathrm{ml} / \mathrm{min}$, $=254 \mathrm{~nm}, t_{\mathrm{R}}=6.6 \mathrm{~min}$, minor; $t_{\mathrm{R}}=7.7 \mathrm{~min}$, major). 


\section{(2S,3R)-3-(tert-Butyldimethylsilyloxy)-2,3-diphenylpropan-1-ol (13c)}<smiles>COC(c1ccccc1)[C@H](CO)c1ccccc1</smiles>

A solution of $\mathrm{Rh}_{2}(\mathrm{OOct})_{4}(3.9 \mathrm{mg}, 0.005 \mathrm{mmol}, 0.01$ eq.), compound 6c (111 mg, 0.5 mmol, 1 eq.) and 2,2-dimethylbutane (5 mL) was heated under reflux for $30 \mathrm{~min}$ and then allowed to cool to room temperature. A solution of compound 11 (262 mg, $1.0 \mathrm{mmol}, 2$ eq.) in 2,2-dimethylbutane (10 mL) was added via syringe pump at a rate of $3.33 \mathrm{ml} / \mathrm{h}$. After addition was complete, the reaction mixture was stirred for $1 \mathrm{~h}$, and then cooled to $0{ }^{\circ} \mathrm{C}$. Diisobutylaluminium hydride (1.0M in toluene, $3.0 \mathrm{ml}, 3.0 \mathrm{mmol}, 6$ eq.) was added, and the reaction stirred for $2 \mathrm{~h}$ at $0{ }^{\circ} \mathrm{C}$ and then $10 \mathrm{~h}$ at room temperature. The reaction mixture was then poured into a 1:1 mix $(50 \mathrm{~mL})$ of $\mathrm{Et}_{2} \mathrm{O}$ and saturated sodium potassium tartrate solution, and the biphasic mixture stirred for $2 \mathrm{~h}$. The layers were separated, and the aqueous layer was extracted with 2 portions $\mathrm{Et}_{2} \mathrm{O}$. The combined organic fractions were dried $\left(\mathrm{Na}_{2} \mathrm{SO}_{4}\right)$ and concentrated in vacuo. Diastereoselectivity $(88 \%$ de) was determined by ${ }^{1} \mathrm{H}$ NMR of the crude reaction mixture, and the product purified by flash chromatography on silica gel using 10:1 hexane/ $\mathrm{Et}_{2} \mathrm{O}$ as eluant to isolate a mixture of the anti and syn isomers as a colorless oil, $42 \mathrm{mg}$; and the pure syn isomer as a colorless oil, $74 \mathrm{mg}$ (total yield: 69\%). [_] $\left.\mathrm{CDCl}_{3}\right):{ }_{7} .26-7.21(\mathrm{~m}, 6 \mathrm{H}), 7.10-7.05(\mathrm{~m}, 4 \mathrm{H}), 4.96(\mathrm{~d}, 1 \mathrm{H}, J=7.0 \mathrm{~Hz}), 3.88(\mathrm{dd}, 1 \mathrm{H}$, $J=11.0,7.5 \mathrm{~Hz}), 3.80$ (dd, 1H, $J=11.0,7.0 \mathrm{~Hz}), 3.15$ (dd, $J=13.0,7.0 \mathrm{~Hz}), 1.79$ (br s, $1 \mathrm{H})$, $0.81(\mathrm{~s}, 9 \mathrm{H}),-0.09$ (s, 3H), -0.27 (s, 3H). ${ }^{13} \mathrm{C}$ NMR $\left(75 \mathrm{MHz}, \mathrm{CDCl}_{3}\right):_{-}$142.4, 139.0, 129.3, 128.0, 127.7, 127.3, 126.8, 126.7, 77.1, 63.7, 56.5, 25.7, 18.0, -4.8, -5.5. IR (film): 3401, 2927, 2857, 1604, 1495, 1471, 1456, 1255, $1071 \mathrm{~cm}^{-1} . m / z$ (ESI): $365\left(\mathrm{MNa}^{+}\right.$, $100 \%$ ), 153 (72\%). Anal calcd. for $\mathrm{C}_{21} \mathrm{H}_{30} \mathrm{O}_{2} \mathrm{Si}$ : C, 73.63; H, 8.83. Found: C, 73.69; $\mathrm{H}$, 8.98. HPLC analysis: $84 \%$ ee $\left(R, R\right.$-Whelk-O1, $3 \% i-\mathrm{PrOH}$ in hexane, $1.0 \mathrm{ml} / \mathrm{min},{ }_{-}=254$ $\mathrm{nm}, t_{\mathrm{R}}=6.5 \mathrm{~min}$, minor; $t_{\mathrm{R}}=7.4 \mathrm{~min}$, major). 
(2S,3R)-3-(tert-Butyldimethylsilyloxy)-3-(4-chlorophenyl)-2-phenylpropan-1-ol (13d)<smiles>[B-]O[C@H](c1ccc(Cl)cc1)[C@H](CO)c1ccccc1</smiles>

A solution of $\mathrm{Rh}_{2}(\mathrm{OOct})_{4}(3.9 \mathrm{mg}, 0.005 \mathrm{mmol}, 0.01$ eq.), compound $\mathbf{6 d}$ (128 mg, 0.5 mmol, 1 eq.) and 2,2-dimethylbutane $(5 \mathrm{~mL})$ was heated under reflux for $30 \mathrm{~min}$ and then allowed to cool to room temperature. A solution of compound 11 (262 mg, $1.0 \mathrm{mmol}, 2$ eq.) in 2,2-dimethylbutane (10 mL) was added via syringe pump at a rate of $3.33 \mathrm{ml} / \mathrm{h}$. After addition was complete, the reaction mixture was stirred for $1 \mathrm{~h}$, and then cooled to $0{ }^{\circ} \mathrm{C}$. After addition was complete, the reaction mixture was stirred for $1 \mathrm{~h}$, and then cooled to $0{ }^{\circ} \mathrm{C}$. Diisobutylaluminium hydride (1.0M in toluene, $3.0 \mathrm{ml}, 3.0 \mathrm{mmol}, 6 \mathrm{eq}$.) was added, and the reaction stirred for $2 \mathrm{~h}$ at $0{ }^{\circ} \mathrm{C}$ and then $10 \mathrm{~h}$ at room temperature. The reaction mixture was then poured into a 1:1 mix $(50 \mathrm{~mL})$ of $\mathrm{Et}_{2} \mathrm{O}$ and saturated sodium potassium tartrate solution. The biphasic mixture was stirred for $2 \mathrm{~h}$. The layers were separated, and the aqueous layer was extracted with 2 portions $\mathrm{Et}_{2} \mathrm{O}$. The combined organic fractions were dried $\left(\mathrm{Na}_{2} \mathrm{SO}_{4}\right)$ and concentrated in vacuo. Diastereoselectivity ( $88 \%$ de) was determined by ${ }^{1} \mathrm{H}$ NMR of the crude reaction mixture, and the product purified by flash chromatography on silica gel using 10:1 hexane/ $\mathrm{Et}_{2} \mathrm{O}$ as eluant to isolate the anti isomer as a colorless oil, $6 \mathrm{mg}$, a mixture of the anti and syn isomers as a colorless oil, $64 \mathrm{mg}$; and the pure syn isomer as a colorless oil, $102 \mathrm{mg}$. (total yield: 91\%). [ ] $]_{\mathrm{D}}+54.1$ (ee 82\%) (c 1.07, $\left.\mathrm{CHCl}_{3}\right) .{ }^{1} \mathrm{H} \mathrm{NMR}\left(500 \mathrm{MHz}, \mathrm{CDCl}_{3}\right):$ _ 7.28-7.21 (m, $3 \mathrm{H}), 7.19$ (d, 2H, J=9.0 Hz), 7.06-7.03 (m, 2H), 6.98 (d, 2H, J=8.5 Hz), 4.99 (d, 1H, $J=7.0 \mathrm{~Hz}), 3.91(\mathrm{dd}, 1 \mathrm{H}, J=10.5,7.5 \mathrm{~Hz}), 3.81$ (dd, 1H, $J=10.5,7.0 \mathrm{~Hz}), 3.08$ (dd, 1H, $J=12.0,7.0 \mathrm{~Hz}$ ), 1.82 (br s, $1 \mathrm{H}), 0.83$ (s, 9H), -0.05 (s, 3H), -0.25 (s, 3H). ${ }^{13} \mathrm{C}$ NMR (125 $\left.\mathrm{MHz}, \mathrm{CDCl}_{3}\right): \_141.0,138.2,132.8,129.4,128.1,128.0,127.9,127.0,76.0,63.5,56.2$, 25.7, 18.0, -4.7, -5.5. IR (film): 3392, 2929, 2856, 1491, 1256, 1089, $837 \mathrm{~cm}^{-1}$. Anal Calcd. for $\mathrm{C}_{21} \mathrm{H}_{29} \mathrm{ClO}_{2} \mathrm{Si}$ : C, 66.90; H, 7.75. Found: C, 66.65; H, 7.82. HPLC analysis: $82 \%$ ee $\left(R, R\right.$-Whelk-O1, $3 \% i$-PrOH in hexane, $1.0 \mathrm{ml} / \mathrm{min}$, $=254 \mathrm{~nm}, t_{\mathrm{R}}=7.4 \mathrm{~min}$, minor; $t_{\mathrm{R}}=8.4$ min, major). 
(2S,3R)-3-(tert-Butyldimethylsilyloxy)-2-phenyl-3-(4(trifluoromethyl)phenyl)propan-1-ol (13e)<smiles>OC[C@H](c1ccccc1)[C@@H]([OH2+])c1ccc(C(F)(F)F)cc1</smiles>

A solution of $\mathrm{Rh}_{2}(\mathrm{OOct})_{4}(3.9 \mathrm{mg}, 0.005 \mathrm{mmol}, 0.01$ eq.), compound 6e (145 mg, 0.5 mmol, 1 eq.) and 2,2-dimethylbutane ( $5 \mathrm{~mL}$ ) was heated under reflux for $45 \mathrm{~min}$, and then allowed to cool to room temperature. A solution of compound 11 (262 $\mathrm{mg}, 1.0$ mmol, 2 eq.) in 2,2-dimethylbutane $(10 \mathrm{~mL})$ was added via syringe pump at a rate of 3.33 $\mathrm{ml} / \mathrm{h}$. After addition was complete, the reaction mixture was stirred for $1 \mathrm{~h}$, and then cooled to $0{ }^{\circ} \mathrm{C}$. Diisobutylaluminium hydride (1.0M in toluene, $3.0 \mathrm{ml}, 3.0 \mathrm{mmol}, 6$ eq.) was added, and the reaction stirred for $2 \mathrm{~h}$ at $0{ }^{\circ} \mathrm{C}$ and then $10 \mathrm{~h}$ at room temperature. The reaction mixture was then poured into a 1:1 mix $(50 \mathrm{~mL})$ of $\mathrm{Et}_{2} \mathrm{O}$ and saturated sodium potassium tartrate solution, and the biphasic mixture stirred for $2 \mathrm{~h}$. The layers were separated, and the aqueous layer was extracted with 2 portions $\mathrm{Et}_{2} \mathrm{O}$. The combined organic fractions were dried $\left(\mathrm{Na}_{2} \mathrm{SO}_{4}\right)$ and concentrated in vacuo. Diastereoselectivity ( $88 \%$ de) was determined by ${ }^{1} \mathrm{H}$ NMR of the crude reaction mixture, and the product purified by flash chromatography on silica gel using 10:1 hexane/Et $\mathrm{Et}_{2} \mathrm{O}$ as eluant to isolate a mixture of the anti and syn isomers as a pale yellow oil, $13 \mathrm{mg}$; and the pure syn isomer as a pale yellow oil, $131 \mathrm{mg}$. Total mass: $144 \mathrm{mg}(70 \%)$. [_] $]_{\mathrm{D}}+54.2$ (ee 85\%) (c 1.51, $\left.\mathrm{CHCl}_{3}\right) .{ }^{1} \mathrm{H} \mathrm{NMR}\left(500 \mathrm{MHz}, \mathrm{CDCl}_{3}\right)$ : 7.46 (d, 2H, J=8.0 Hz), 7.28-7.19 (m, 3H), 7.15 (d, 2H, $J=8.0 \mathrm{~Hz}), 7.06-6.99(\mathrm{~m}, 2 \mathrm{H}), 5.10$ (d, 1H, $J=7.0 \mathrm{~Hz}), 3.91$ (dd, 1H, $J=11.0,7.0$ $\mathrm{Hz}), 3.82$ (dd, 1H, $J=11.0,7.0 \mathrm{~Hz}), 3.08$ (dd, 1H, $J=12.0,7.0 \mathrm{~Hz}), 1.87$ (br s, 1H), 0.85 (s, $9 \mathrm{H}),-0.03(\mathrm{~s}, 3 \mathrm{H}),-0.26(\mathrm{~s}, 3 \mathrm{H}) .{ }^{13} \mathrm{C} \mathrm{NMR}\left(125 \mathrm{MHz}, \mathrm{CDCl}_{3}\right)$ :_146.7, 137.9, 129.4, 129.3 (q, $J=32.0 \mathrm{~Hz}), 128.0,127.1,126.9,124.6$ (q, $J=4.0 \mathrm{~Hz}), 124.2$ (q, $J=272.5 \mathrm{~Hz}$ ), 75.7 (2C), 56.3, 25.7, 18.0, -4.8, -5.5. IR (film): 3401, 2938, 2878, 1620, 1472, 1326, $1018 \mathrm{~cm}^{-1} . \mathrm{m} / z(\mathrm{CI}): 411\left(\mathrm{MH}^{+}, 3 \%\right), 353$ (10\%), 279 (68\%), 235 (34\%), 133 (100\%). HRMS calcd. for $\mathrm{C}_{22} \mathrm{H}_{29} \mathrm{O}_{2} \mathrm{~F}_{3} \mathrm{NaSi}\left(\mathrm{MNa}^{+}\right)$: 433.1781. Found: 433.1793. HPLC analysis: $85 \%$ ee $\left(R, R\right.$-Whelk-O1, $1 \% i$-PrOH in hexane, $1.0 \mathrm{ml} / \mathrm{min},=254 \mathrm{~nm}, t_{\mathrm{R}}=10.4 \mathrm{~min}$, minor; $t_{\mathrm{R}}=11.5 \mathrm{~min}$, major). 


\section{(S)-Ethyl 2-(2-(4-bromophenyl)acetoxy)propanoate}<smiles>CCOC(=O)[C@H](C)OC(=O)Cc1ccc(Br)cc1</smiles>

1,3-Dicyclohexylcarbodiimide (1.0 $\mathrm{M}$ in $\mathrm{CH}_{2} \mathrm{Cl}_{2}, 10 \mathrm{~mL}, 10 \mathrm{mmol}, 1$ eq.) was added dropwise via syringe to a cold $\left(0{ }^{\circ} \mathrm{C}\right)$ solution of 4-bromophenylacetic acid $(2.15 \mathrm{~g}, 10$ mmol, 1 eq.), $N, N$-dimethylaminopyridine (122 mg, $1 \mathrm{mmol}, 0.1$ eq.) and ethyl (S)-lactate (2.27 mL, $20 \mathrm{mmol}, 2$ eq.) in $\mathrm{CH}_{2} \mathrm{Cl}_{2}(50 \mathrm{~mL})$. A white precipitate began to form, and the reaction mixture was allowed to reach room temperature over $12 \mathrm{~h}$. The suspension was then filtered through Celite ${ }^{\circledR}$, and washed with $\mathrm{CH}_{2} \mathrm{Cl}_{2}$. The filtrate was concentrated in vacuo, and purified by chromatography on silica gel using 7:1 hexane/Et $\mathrm{t}_{2} \mathrm{O}$ as eluant to isolate a pale yellow oil, $3.02 \mathrm{~g} \mathrm{(96 \% ).} \mathrm{[} \mathrm{]}]_{\mathrm{D}}-23.8\left(c\right.$ 1.82, $\left.\mathrm{CHCl}_{3}\right) .{ }^{1} \mathrm{H} \mathrm{NMR}(500 \mathrm{MHz}$, $\left.\mathrm{CDCl}_{3}\right):{ }_{-} .46(\mathrm{~d}, 2 \mathrm{H}, J=8.0 \mathrm{~Hz}), 7.20(\mathrm{~d}, 2 \mathrm{H}, J=8.0 \mathrm{~Hz}), 5.09(\mathrm{q}, 1 \mathrm{H}, J=7.0 \mathrm{~Hz}), 4.18(\mathrm{q}$, $2 \mathrm{H}, J=7.0 \mathrm{~Hz}), 3.71-3.63(\mathrm{~m}, 2 \mathrm{H}), 1.49$ (d, 3H, $J=7.0 \mathrm{~Hz}), 1.24$ (t, 3H, $J=7.0 \mathrm{~Hz}) .{ }^{13} \mathrm{C}$ NMR (125 MHz, $\left.\mathrm{CDCl}_{3}\right)$ : _ 170.3, 170.2, 132.4, 131.4, 130.9, 121.0, 68.9, 61.2, 40.0, 16.6, 13.8. IR (film): 2987, 2939, 1741, 1490, 1211, $1097 \mathrm{~cm}^{-1} . \mathrm{m} / z$ (ESI): $337\left(\mathrm{MNa}^{+}\right.$, 24\%), 225 (25\%), 207 (25\%). HRMS Calcd. for $\mathrm{C}_{13} \mathrm{H}_{15} \mathrm{O}_{4} \mathrm{BrNa}\left(\mathrm{MNa}^{+}\right)$: 337.0046. Found: 337.0042 .

(S)-2-[2-(4-Bromo-phenyl)-2-diazo-acetoxy]-propionic acid ethyl ester<smiles>CCOC(=O)[C@H](C)OC(=O)C(=N)c1ccc(Br)cc1</smiles>

A solution of (S)-ethyl 2-(2-(4-bromophenyl)acetoxy)propanoate (2.54 g, $8.06 \mathrm{mmol}, 1.0$ eq.) and para-acetamidobenzensulfonyl azide (2.03 g, $8.87 \mathrm{mmol}, 1.1$ eq.) in MeCN (30 $\mathrm{mL})$ was cooled to $0{ }^{\circ} \mathrm{C}$ and 1,8-diazabicyclo[5.4.0]undec-7-ene (1.08 mL, $7.25 \mathrm{mmol}$, 0.9 eq.) was added dropwise via syringe. The reaction mixture was allowed to reach room temperature over $12 \mathrm{~h}$. The reaction was quenched by addition of saturated $\mathrm{NH}_{4} \mathrm{Cl}$ solution, and extracted into $\mathrm{Et}_{2} \mathrm{O}(3 \mathrm{x})$. The combined organic fractions were dried $\left(\mathrm{Na}_{2} \mathrm{SO}_{4}\right)$ and concentrated in vacuo. The residue was triturated with 1:1 hexane/Et $\mathrm{E}_{2} \mathrm{O}$, and filtered through Celite ${ }^{\circledR}$. The filtrate was concentrated in vacuo and purified by flash chromatography on silica gel using 8:1 hexane/ $\mathrm{Et}_{2} \mathrm{O}$ as eluant to isolate an orange solid, 
$2.65 \mathrm{~g}(96 \%)$. [ $]_{\mathrm{D}}+4.2\left(\mathrm{c} 1.51, \mathrm{CHCl}_{3}\right) .{ }^{1} \mathrm{H}$ NMR $\left(400 \mathrm{MHz}, \mathrm{CDCl}_{3}\right): \_7.50(\mathrm{~d}, 2 \mathrm{H}$, $J=8.5 \mathrm{~Hz}), 7.37$ (d, 2H, $J=8.5 \mathrm{~Hz}), 5.25$ (q, 1H, $J=7.0 \mathrm{~Hz}), 4.24$ (q, 2H, $J=7.0 \mathrm{~Hz}), 1.56$ (d, $3 \mathrm{H}, J=7.0 \mathrm{~Hz}), 1.30$ (t, $3 \mathrm{H}, J=7.0 \mathrm{~Hz}) .{ }^{13} \mathrm{C} \mathrm{NMR}\left(75 \mathrm{MHz}, \mathrm{CDCl}_{3}\right): \_170.5,164.0$, $131.9,125.3,124.3,119.4,69.1,61.5,16.9,14.0$. The carbon attached to the diazo moiety was not observed. IR $\left(\mathrm{CH}_{2} \mathrm{Cl}_{2}\right): 2985,2093,1754,1708,1492,1209,1157,1096$ $\mathrm{cm}^{-1} . \mathrm{m} / z$ (EI): $340\left(\mathrm{M}^{+}, 9 \%\right), 199$ (13\%), 185 (98\%), 183 (100\%). HRMS Calcd. for $\mathrm{C}_{13} \mathrm{H}_{13} \mathrm{O}_{4} \mathrm{~N}_{2} \mathrm{Br}: 340.0053$. Found: 340.0044 .

\section{(S)-Ethyl 2-(2-p-tolylacetoxy)propanoate}<smiles>CCOC(=O)C(C)OC(=O)Cc1ccc(C)cc1</smiles>

1,3-Dicyclohexylcarbodiimide (1.0 M in $\mathrm{CH}_{2} \mathrm{Cl}_{2}, 10 \mathrm{~mL}, 10 \mathrm{mmol}, 1$ eq.) was added dropwise via syringe to a cold $\left(0{ }^{\circ} \mathrm{C}\right)$ solution of $p$-tolylacetic acid $(1.50 \mathrm{~g}, 10 \mathrm{mmol}, 1$ eq.), $N, N$-dimethylaminopyridine (122 mg, $1 \mathrm{mmol}, 0.1$ eq.) and ethyl (S)-lactate (2.27 $\mathrm{mL}, 20 \mathrm{mmol}, 2$ eq.) in $\mathrm{CH}_{2} \mathrm{Cl}_{2}(50 \mathrm{~mL})$. A white precipitate began to form. The reaction mixture was allowed to reach room temperature over $12 \mathrm{~h}$. The suspension was then filtered through Celite ${ }^{\circledR}$, and washed with $\mathrm{CH}_{2} \mathrm{Cl}_{2}$. The filtrate was concentrated in vacuo, and purified by chromatography on silica gel using 7:1 hexane/Et $\mathrm{t}_{2} \mathrm{O}$ as eluant to isolate a colorless oil, $2.40 \mathrm{~g}(96 \%)$. [ $]_{\mathrm{D}}-35.7\left(c \quad 1.72, \mathrm{CHCl}_{3}\right) .{ }^{1} \mathrm{H}$ NMR $\left(500 \mathrm{MHz}, \mathrm{CDCl}_{3}\right)$ : _ 7.20 (d, 2H, $J=8.0 \mathrm{~Hz}), 7.14$ (d, 2H, $J=8.0 \mathrm{~Hz}), 5.09$ (q, 1H, $J=7.0 \mathrm{~Hz}), 4.18$ (q, 2H, $J=7.0 \mathrm{~Hz}$ ), 3.71-3.63 (m, 2H), 2.34 (s, 3H), 1.49 (d, 3H, $J=7.0 \mathrm{~Hz}), 1.24$ (t, 3H, $J=7.0$ $\mathrm{Hz}) .{ }^{13} \mathrm{C}$ NMR $\left(125 \mathrm{MHz}, \mathrm{CDCl}_{3}\right)$ :_170.8, 170.3, 136.3, 130.3, 128.92, 128.90, 68.7, 60.9, 40.1, 20.7, 16.5, 13.7. IR (film): 2988, 2941, 1741, 1517, 1207, $1098 \mathrm{~cm}^{-1} . \mathrm{m} / z$ (ESI): $273\left(\mathrm{MNa}^{+}, 22 \%\right), 268(100 \%), 251(30 \%)$. HRMS Calcd. for $\mathrm{C}_{14} \mathrm{H}_{18} \mathrm{O}_{4} \mathrm{Na}$ $\left(\mathrm{MNa}^{+}\right): 273.1097$. Found: 273.1096.

\section{(S)-2-(2-Diazo-2-p-tolyl-acetoxy)-propionic acid ethyl ester}

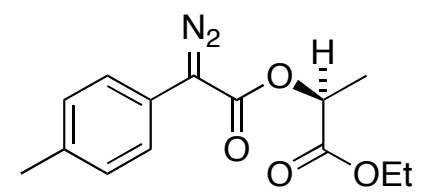

A solution of 2-(2-p-tolyl-acetoxy)-propionic acid ethyl ester (2.03 g, $8.11 \mathrm{mmol}, 1.0$ eq.) and para-acetamidobenzensulfonyl azide $(2.04 \mathrm{~g}, 8.92 \mathrm{mmol}, 1.1 \mathrm{eq}$.) in acetonitrile (30 $\mathrm{mL}$ ) was cooled to $0{ }^{\circ} \mathrm{C}$ and 1,8-diazabicyclo[5.4.0]undec-7-ene $(1.09 \mathrm{~mL}, 7.30 \mathrm{mmol}$, 
0.9 eq.) was added dropwise via syringe. The reaction mixture was allowed to reach room temperature over $12 \mathrm{~h}$. The reaction was quenched by addition of saturated $\mathrm{NH}_{4} \mathrm{Cl}$ solution, and extracted into $\mathrm{Et}_{2} \mathrm{O}(3 \mathrm{x})$. The combined organic fractions were dried $\left(\mathrm{Na}_{2} \mathrm{SO}_{4}\right)$ and concentrated in vacuo. The residue was triturated with $1: 1$ hexane/Et $2 \mathrm{O}$, and filtered through Celite ${ }^{\circledR}$. The filtrate was concentrated in vacuo and purified by flash chromatography on silica gel using 8:1 hexane/ $\mathrm{Et}_{2} \mathrm{O}$ as eluant to isolate an orange solid, $2.06 \mathrm{~g}(92 \%)$. [ $]_{\mathrm{D}}+2.1$ (c 1.63, $\left.\mathrm{CHCl}_{3}\right) .{ }^{1} \mathrm{H}$ NMR $\left(500 \mathrm{MHz}, \mathrm{CDCl}_{3}\right)$ : 7.37 (d, 2H, $J=8.0 \mathrm{~Hz}), 7.21(\mathrm{~d}, 2 \mathrm{H}, J=8.0 \mathrm{~Hz}), 5.26(\mathrm{q}, 1 \mathrm{H}, J=7.0 \mathrm{~Hz}), 4.24(\mathrm{q}, 2 \mathrm{H}, J=7.0 \mathrm{~Hz}), 2.35$ (s, 3H), 1.55 (d, 3H, $J=7.0 \mathrm{~Hz}), 1.30(\mathrm{t}, 3 \mathrm{H}, J=7.0 \mathrm{~Hz}) .{ }^{13} \mathrm{C} \mathrm{NMR}\left(75 \mathrm{MHz}, \mathrm{CDCl}_{3}\right)$ : _ 170.7, 164.7, 135.9, 129.6, 124.2, 121.8, 68.9, 61.4, 21.0, 17.0, 14.1. The carbon attached to the diazo moiety was not observed. IR $\left(\mathrm{CH}_{2} \mathrm{Cl}_{2}\right): 2986,2090,1753,1708$, 1516, 1450, 1249, 1155, $1097 \mathrm{~cm}^{-1} . \mathrm{m} / z$ (EI): $276\left(\mathrm{M}^{+}, 9 \%\right), 119$ (100\%). Anal calcd. for $\mathrm{C}_{14} \mathrm{H}_{16} \mathrm{O}_{4} \mathrm{~N}_{2}$ : C, 60.86; H, 5.84; N, 10.14. Found: C, 60.89; H, 5.93, N, 9.89.

\section{(S)-Ethyl 2-(2-(4-methoxyphenyl)acetoxy)propanoate)}

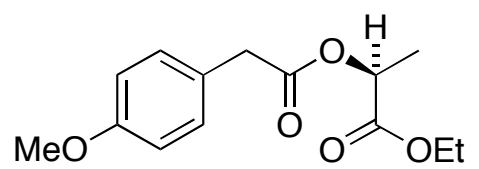

1,3-Dicyclohexylcarbodiimide (1.0 M in $\mathrm{CH}_{2} \mathrm{Cl}_{2}, 10 \mathrm{~mL}, 10 \mathrm{mmol}, 1$ eq.) was added dropwise via syringe to a cold $\left(0{ }^{\circ} \mathrm{C}\right)$ solution of 4-methoxyphenylacetic acid $(1.66 \mathrm{~g}, 10$ mmol, 1 eq.), $N, N$-dimethylaminopyridine (122 mg, $1 \mathrm{mmol}, 0.1$ eq.) and ethyl (S)-lactate (2.27 mL, $20 \mathrm{mmol}, 2$ eq.) in $\mathrm{CH}_{2} \mathrm{Cl}_{2}(50 \mathrm{~mL})$ was cooled to $0{ }^{\circ} \mathrm{C}$. A white precipitate began to form, and the reaction mixture was allowed to reach room temperature over $12 \mathrm{~h}$. The suspension was then filtered through Celite ${ }^{\circledR}$, and washed with $\mathrm{CH}_{2} \mathrm{Cl}_{2}$. The filtrate was concentrated in vacuo, and purified by chromatography on silica gel using 7:1 hexane/ $\mathrm{Et}_{2} \mathrm{O}$ as eluant to isolate a colorless oil, $2.13 \mathrm{~g}(80 \%)$. [ $\left.]\right]_{\mathrm{D}}-39.7$ (c 2.34, $\mathrm{CHCl}_{3}$ ). ${ }^{1} \mathrm{H}$ NMR (500 MHz, CDCl $)$ : _ 7.23 (d, 2H, $\left.J=8.5 \mathrm{~Hz}\right), 6.87$ (d, 2H, J=8.5 Hz), 5.08 (q, 1H, $J=7.0 \mathrm{~Hz}$ ), 4.18 (q, 2H, J=7.5 Hz), 3.80 (s, 3H), 3.87-3.61 (m, 2H), 1.49 (d, 3H, $J=7.0 \mathrm{~Hz}), 1.24$ (t, $3 \mathrm{H}, J=7.0 \mathrm{~Hz}) .{ }^{13} \mathrm{C}$ NMR $\left(75 \mathrm{MHz}, \mathrm{CDCl}_{3}\right)$ :_171.1, 170.5, 158.6, 130.2, 125.5, 113.8, 68.8, 61.1, 55.0, 39.8, 16.7, 13.9. IR (film): 2988, 1743, 1614, 1515, 1463, 1250, 1154, $1098 \mathrm{~cm}^{-1} . \mathrm{m} / z$ (ESI): $289\left(\mathrm{MNa}^{+}, 16 \%\right), 284$ (100\%), $267(23 \%)$. Anal. calcd. for $\mathrm{C}_{14} \mathrm{H}_{18} \mathrm{O}_{5}$ : C, 63.15; H, 6.81. Found: $\mathrm{C}, 63.05 ; \mathrm{H}, 7.05$. 


\section{(S)-2-[2-Diazo-2-(4-methoxy-phenyl)-acetoxy]-propionic acid ethyl ester}

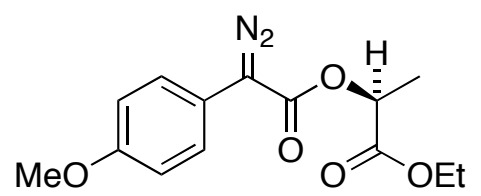

A solution of $(S)$-ethyl 2-(2-(4-methoxyphenyl)acetoxy)propanoate) (1.79 g, $6.74 \mathrm{mmol}$, 1.0 eq.) and para-acetamidobenzensulfonyl azide (1.69 g, $7.41 \mathrm{mmol}, 1.1$ eq.) in acetonitrile $(30 \mathrm{~mL})$ was cooled to $0{ }^{\circ} \mathrm{C}$ and 1,8-diazabicyclo[5.4.0]undec-7-ene $(0.91$ $\mathrm{mL}, 6.06 \mathrm{mmol}, 0.9$ eq.) was added dropwise via syringe. The reaction mixture was allowed to reach room temperature over $12 \mathrm{~h}$. The reaction was quenched by addition of saturated $\mathrm{NH}_{4} \mathrm{Cl}$ solution, and extracted into $\mathrm{Et}_{2} \mathrm{O}(3 \mathrm{x})$. The combined organic fractions were dried $\left(\mathrm{Na}_{2} \mathrm{SO}_{4}\right)$ and concentrated in vacuo. The residue was triturated with 1:1 hexane/Et $2 \mathrm{O}$, and filtered through Celite ${ }^{\circledR}$. The filtrate was concentrated in vacuo and purified by flash chromatography on silica gel using 8:1 hexane/Et ${ }_{2} \mathrm{O}$ as eluant to isolate an orange solid, $1.70 \mathrm{~g}(86 \%)$. [ ] $]_{\mathrm{D}}-8.8\left(\mathrm{c} 1.02, \mathrm{CHCl}_{3}\right) .{ }^{1} \mathrm{H} \mathrm{NMR}\left(500 \mathrm{MHz}, \mathrm{CDCl}_{3}\right)$ : 7.39 (d, 2H, J=8.5 Hz), 6.95 (d, 2H, J=8.5 Hz), 5.25 (q, 1H, J=7.0 Hz), 4.24 (q, 2H, $J=7.0 \mathrm{~Hz}), 3.82(\mathrm{~s}, 3 \mathrm{H}), 1.55(\mathrm{~d}, 3 \mathrm{H}, J=7.0 \mathrm{~Hz}), 1.30(\mathrm{t}, 3 \mathrm{H}, J=7.0 \mathrm{~Hz}) .{ }^{13} \mathrm{C} \mathrm{NMR}(75$ $\mathrm{MHz}_{\mathrm{CDCl}} \mathrm{CD}_{2}$ _ $170.7,165.0,158.2,126.1,116.6,114.6,68.9,61.4,55.3,17.0,14.1$. The carbon attached to the diazo moiety was not observed. IR $\left(\mathrm{CH}_{2} \mathrm{Cl}_{2}\right): 2986,2089,1747$, 1708, 1610, 1515, 1254, 1175, $1096 \mathrm{~cm}^{-1} \cdot \mathrm{m} / z(\mathrm{EI}): 292\left(\mathrm{M}^{+}, 3 \%\right), 148(28 \%), 135$ (100\%). HRMS Calcd. for $\mathrm{C}_{14} \mathrm{H}_{16} \mathrm{O}_{5} \mathrm{~N}_{2}\left(\mathrm{M}^{+}\right)$: 292.1054. Found: 292.1055 .

\section{(S)-Ethyl 2-(2-(4-(trifluoromethyl)phenyl)acetoxy)propanoate}<smiles>CCOC(=O)[C@H](C)OC(=O)Cc1ccc(C(F)(F)F)cc1</smiles>

1,3-Dicyclohexylcarbodiimide (1.0 M in $\mathrm{CH}_{2} \mathrm{Cl}_{2}, 4.90 \mathrm{~mL}, 4.90 \mathrm{mmol}, 1$ eq.) was added dropwise via syringe to a cold $\left(0{ }^{\circ} \mathrm{C}\right)$ solution of 4-(trifluoromethyl)phenylacetic acid (1.00 g, $4.90 \mathrm{mmol}, 1$ eq.), $N, N$-dimethylaminopyridine (60 mg, $0.49 \mathrm{mmol}, 0.1$ eq.) and ethyl $(S)$-lactate $\left(1.11 \mathrm{~mL}, 9.80 \mathrm{mmol}, 2\right.$ eq.) in $\mathrm{CH}_{2} \mathrm{Cl}_{2}(30 \mathrm{~mL})$. A white precipitate began to form, and the reaction mixture was allowed to reach room temperature over $12 \mathrm{~h}$. The suspension was then filtered through Celite ${ }^{\circledR}$, and washed with $\mathrm{CH}_{2} \mathrm{Cl}_{2}$. The filtrate was concentrated in vacuo, and purified by chromatography on silica gel using 8:1 hexane/ $\mathrm{Et}_{2} \mathrm{O}$ as eluant to isolate a colorless oil, $1.45 \mathrm{~g}(98 \%)$. [ $]_{\mathrm{D}}-25.9\left(\mathrm{c} 1.88, \mathrm{CHCl}_{3}\right)$. 
${ }^{1} \mathrm{H}$ NMR $\left(500 \mathrm{MHz}, \mathrm{CDCl}_{3}\right)$ : 7.60 (d, 2H, $\left.J=8.0 \mathrm{~Hz}\right), 7.45$ (d, 2H, J=8.0 Hz), 5.10 (q, $1 \mathrm{H}, J=7.0 \mathrm{~Hz}$ ), 4.18 (q, 2H, $J=7.0 \mathrm{~Hz}), 3.82-3.74$ (m, 2H), 1.50 (d, 3H, $J=7.0 \mathrm{~Hz}), 1.24$ (t, $3 \mathrm{H}, J=7.0 \mathrm{~Hz}) .{ }^{13} \mathrm{C} \mathrm{NMR}\left(125 \mathrm{MHz}, \mathrm{CDCl}_{3}\right): 170.4,170.2,137.5,129.7,129.5$ (q, $J=32.5 \mathrm{~Hz}), 125.4$ (q, $J=4.0 \mathrm{~Hz}), 124.1$ (q, $J=272.0), 69.2,61.4,40.5,16.8,13.9$. IR (film): 2991, 2943, 1745, 1621, 1451, 1328, 1164, 1128, 1068, $1021 \mathrm{~cm}^{-1} \cdot m / z$ (ESI): 327 $\left(\mathrm{MNa}^{+}, 41 \%\right), 279$ (100\%). Anal. Calcd. for $\mathrm{C}_{14} \mathrm{H}_{15} \mathrm{O}_{4} \mathrm{~F}_{3}: \mathrm{C}, 55.26$; H, 4.97. Found: $\mathrm{C}$, 55.54; H, 5.06.

\section{(S)-2-[2-Diazo-2-(4-trifluoromethyl-phenyl)-acetoxy]-propionic acid ethyl ester}<smiles>CCOC(=O)[C@H](C)OC(=O)C(=N)c1ccc(C(F)(F)F)cc1</smiles>

A solution of (S)-ethyl 2-(2-(4-(trifluoromethyl)phenyl)acetoxy)propanoate (1.27 g, 4.17 mmol, 1.0 eq.) and para-acetamidobenzensulfonyl azide (1.05 g, $4.59 \mathrm{mmol}, 1.1$ eq.) in acetonitrile $(30 \mathrm{~mL})$ was cooled to $0{ }^{\circ} \mathrm{C}$ and 1,8-diazabicyclo[5.4.0]undec-7-ene $(0.56$ $\mathrm{mL}, 3.76 \mathrm{mmol}, 0.9$ eq.) was added dropwise via syringe. The reaction mixture was slowly allowed to reach room temperature by letting the ice bath melt, and stirred overnight. The reaction was quenched by addition of saturated $\mathrm{NH}_{4} \mathrm{Cl}$ solution, and extracted into $\mathrm{Et}_{2} \mathrm{O}(3 \mathrm{x})$. The combined organic fractions were dried $\left(\mathrm{Na}_{2} \mathrm{SO}_{4}\right)$ and concentrated in vacuo. The residue was triturated with $1: 1$ hexane/ $/ \mathrm{Et}_{2} \mathrm{O}$, and filtered through Celite ${ }^{\circledR}$. The filtrate was concentrated in vacuo and purified by flash chromatography on silica gel using 8:1 hexane/ $\mathrm{Et}_{2} \mathrm{O}$ as eluant to isolate an orange oil, 1.03 g (75\%). [ $]_{\mathrm{D}}+8.4$ (c 1.21, $\left.\mathrm{CHCl}_{3}\right) .{ }^{1} \mathrm{H}$ NMR $\left(500 \mathrm{MHz}, \mathrm{CDCl}_{3}\right)$ : $7.54-7.48$ (m, 4H), 5.17 (q, 1H, J=7.0 Hz), 4.15 (q, 2H, J=7.0 Hz), 1.48 (d, 3H, J=7.0 Hz), 1.20 (t, 3H, $J=7.0 \mathrm{~Hz}) .{ }^{13} \mathrm{C}$ NMR $\left(75 \mathrm{MHz}, \mathrm{CDCl}_{3}\right){ }_{-}$170.3, 163.5, 129.7, 129.4, 127.4 (q, $J=33.0$ Hz), 125.6 (q, $J=4.0 \mathrm{~Hz}), 123.9$ (q, $J=271.5 \mathrm{~Hz}), 69.1,61.4,16.7$, 13.8. The carbon attached to the diazo moiety was not observed. IR $\left(\mathrm{CH}_{2} \mathrm{Cl}_{2}\right): 2989,2099,1754,1712$, 1617, 1328, 1163, 1125, $1072 \mathrm{~cm}^{-1} . \mathrm{m} / z(\mathrm{EI}): 330\left(\mathrm{M}^{+}, 15 \%\right), 274$ (22\%), 173 (100\%), 157 (50\%), 145 (56\%). HRMS Calcd. for $\mathrm{C}_{14} \mathrm{H}_{13} \mathrm{O}_{4} \mathrm{~N}_{2} \mathrm{~F}_{3}: 330.0822$. Found: 330.0824 . 
(2S,3R)-3-(tert-Butyldimethylsilyloxy)-3-(4-methoxyphenyl)-2-p-tolylpropan-1-ol (14)

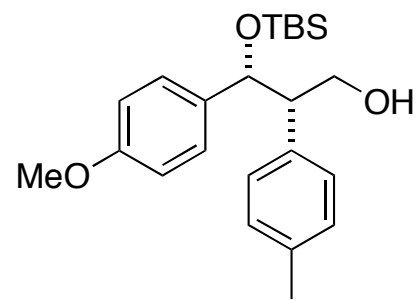

A solution of $\mathrm{Rh}_{2}(\mathrm{OOct})_{4}(3.9 \mathrm{mg}, 0.005 \mathrm{mmol}, 0.01$ eq.), compound $6 \mathbf{a}$ (126 mg, 0.5 mmol, 1 eq.) and 2,2-dimethylbutane ( $5 \mathrm{~mL}$ ) was heated under reflux for $45 \mathrm{~min}$. A solution of 2-(2-diazo-2-p-tolyl-acetoxy)-propionic acid ethyl ester (276 mg, $1.0 \mathrm{mmol}, 2$ eq.) in 2,2-dimethylbutane $(10 \mathrm{~mL})$ was added via syringe pump at a rate of $3.33 \mathrm{ml} / \mathrm{h}$ at $50{ }^{\circ} \mathrm{C}$. After addition was complete, the reaction mixture was stirred for $1 \mathrm{~h}$ at $50{ }^{\circ} \mathrm{C}$, and then cooled to room temperature, and then to $0{ }^{\circ} \mathrm{C}$. Diisobutylaluminium hydride $(1.0 \mathrm{M}$ in toluene, $3.0 \mathrm{ml}, 3.0 \mathrm{mmol}, 6 \mathrm{eq}$.) was added, and the reaction stirred for $2 \mathrm{~h}$ at $0{ }^{\circ} \mathrm{C}$ and then $10 \mathrm{~h}$ at room temperature. The reaction mixture was then poured into a 1:1 mix (50 $\mathrm{mL}$ ) of $\mathrm{Et}_{2} \mathrm{O}$ and saturated sodium potassium tartrate solution, and the biphasic mixture stirred for $2 \mathrm{~h}$. The layers were separated, and the aqueous layer was extracted with 2 portions $\mathrm{Et}_{2} \mathrm{O}$. The combined organic fractions were dried $\left(\mathrm{Na}_{2} \mathrm{SO}_{4}\right)$ and concentrated in vacuo. Diastereoselectivity ( $84 \%$ de) was determined by ${ }^{1} \mathrm{H}$ NMR of the crude reaction mixture, and the product purified by flash chromatography on silica gel using 10:1 hexane $/ \mathrm{Et}_{2} \mathrm{O}$ as eluant to isolate the anti isomer as a colorless oil, $12 \mathrm{mg}$; a mixture of the anti and syn isomers as a colorless oil, $13 \mathrm{mg}$; and the syn isomer as a colorless oil, 100 mg (total yield: 65\%). [ ] $]_{\mathrm{D}}+36.0$ (ee 68\%) $\left(c 1.26, \mathrm{CHCl}_{3}\right) .{ }^{1} \mathrm{H} \mathrm{NMR}\left(500 \mathrm{MHz}, \mathrm{CDCl}_{3}\right)$ : _ 7.05 (d, 2H, $J=7.5 \mathrm{~Hz}), 6.99$ (d, 2H, $J=9.0 \mathrm{~Hz}), 6.93$ (d, 2H, $J=7.5 \mathrm{~Hz}), 6.77$ (d, 2H, $J=9.0 \mathrm{~Hz}$ ), 4.90 (d, 1H, J=6.5 Hz), 3.87 (dd, 1H, $J=11.0,7.5 \mathrm{~Hz}$ ), 3.79 (s, 3H), 3.75 (dd, $1 \mathrm{H}, J=11.0,6.5 \mathrm{~Hz}$ ), 3.11 (dd, 1H, $J=12.5,6.5 \mathrm{~Hz}$ ), 2.32 (s, 3H), 2.03 (br s, 1H), 0.82 (s, 9H), -0.08 (s, 3H), -0.25 (s, 3H). ${ }^{13} \mathrm{C}$ NMR $\left(75 \mathrm{MHz}, \mathrm{CDCl}_{3}\right)$ : _158.6, 136.2, 135.8, 134.3, 129.1, 128.6, 127.8, 112.9, 76.9, 63.7, 55.8, 55.0, 25.6, 20.9, 17.9, -4.9, -5.6. IR (film): 3446, 2360, 2342, 1615, 1506, 1254, $1026 \mathrm{~cm}^{-1} . \mathrm{m} / z$ (ESI): $409\left(\mathrm{MNa}^{+}, 100 \%\right)$, 337 (69\%), 299 (50\%), 298 (20\%). Anal calcd. for $\mathrm{C}_{23} \mathrm{H}_{34} \mathrm{O}_{3} \mathrm{Si}: \mathrm{C}, 71.46$; H, 8.86. Found: $\mathrm{C}, 71.17 ; \mathrm{H}, 8.68$. HPLC analysis: $68 \%$ ee $(R, R$-Whelk-O1, $3 \% i$-PrOH in hexane, 1.0 $\mathrm{ml} / \mathrm{min},=254 \mathrm{~nm}, t_{\mathrm{R}}=10.5 \mathrm{~min}$, minor; $t_{\mathrm{R}}=12.9 \mathrm{~min}$, major). 
(2S,3R)-3-(tert-Butyldimethylsilyloxy)-2,3-bis(4-methoxyphenyl)propan-1-ol (15)

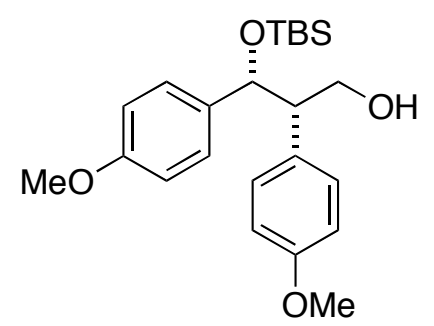

A solution of $\mathrm{Rh}_{2}(\mathrm{OOct})_{4}(3.9 \mathrm{mg}, 0.005 \mathrm{mmol}, 0.01$ eq.), compound 6a (126 mg, 0.5 mmol, 1 eq.) and 2,2-dimethylbutane $(5 \mathrm{~mL})$ was heated under reflux for $45 \mathrm{~min}$. A solution of 2-[2-diazo-2-(4-methoxy-phenyl)-acetoxy]-propionic acid ethyl ester (292 $\mathrm{mg}, 1.0 \mathrm{mmol}, 2$ eq.) in 2,2-dimethylbutane $(20 \mathrm{~mL})$ was added via syringe pump at a rate of $6.8 \mathrm{ml} / \mathrm{h}$ at $50{ }^{\circ} \mathrm{C}$. After addition was complete, the reaction mixture was stirred for $1 \mathrm{~h}$ at $50{ }^{\circ} \mathrm{C}$, and then cooled to room temperature, and then to $0{ }^{\circ} \mathrm{C}$. Diisobutylaluminium hydride (1.0M in toluene, $3.0 \mathrm{ml}, 3.0 \mathrm{mmol}, 6$ eq.) was added, and the reaction stirred for $2 \mathrm{~h}$ at $0{ }^{\circ} \mathrm{C}$ and then $10 \mathrm{~h}$ at room temperature. The reaction mixture was then poured into a 1:1 mix $(50 \mathrm{~mL})$ of $\mathrm{Et}_{2} \mathrm{O}$ and saturated sodium potassium tartrate solution, and the biphasic mixture stirred for $2 \mathrm{~h}$. The layers were separated, and the aqueous layer was extracted with 2 portions $\mathrm{Et}_{2} \mathrm{O}$. The combined organic fractions were dried $\left(\mathrm{Na}_{2} \mathrm{SO}_{4}\right)$ and concentrated in vacuo. Diastereoselectivity $(85 \%$ de) was determined by ${ }^{1} \mathrm{H}$ NMR of the crude reaction mixture, and the product purified by flash chromatography on silica gel using 10:1 hexane/ $\mathrm{Et}_{2} \mathrm{O}$ as eluant to isolate the anti isomer as a pale yellow oil, $4 \mathrm{mg}$, a mixture of the anti and syn isomers as a pale yellow oil, 44 $\mathrm{mg}$; and the pure syn isomer as a colorless oil, $84 \mathrm{mg}$ (total yield: 66\%). [_] $]_{\mathrm{D}}+41.2$ (ee 82\%) (c 1.19, $\left.\mathrm{CHCl}_{3}\right) .{ }^{1} \mathrm{H} \mathrm{NMR}\left(500 \mathrm{MHz}, \mathrm{CDCl}_{3}\right)$ :_6.96 (d, 2H, J=8.5 Hz), 6.95 (d, 2H, $J=8.5 \mathrm{~Hz}), 6.79$ (d, 2H, $J=8.5 \mathrm{~Hz}), 6.76$ (d, 2H, $J=8.5 \mathrm{~Hz}), 4.89$ (d, 1H, J=7.0 Hz), 3.88 (dd, 1H, J=10.5, $7.0 \mathrm{~Hz}), 3.79$ (s, 6H), 3.75 (dd, 1H, J=10.5, $7.0 \mathrm{~Hz}), 3.07$ (dd, 1H, $J=12.5,7.0 \mathrm{~Hz}), 1.99$ (br s, $1 \mathrm{H}), 0.83$ (s, 9H), -0.06 (s, 3H), -0.25 (s, 3H). ${ }^{13} \mathrm{C}$ NMR $(75$ $\left.\mathrm{MHz}, \mathrm{CDCl}_{3}\right): \_158.7,158.4,134.4,130.9,130.3,127.9,113.4,113.0,76.9,63.8,55.4$, 55.2, 55.1, 25.7, 18.0, -4.8, -5.5. IR (film): 3445, 2952, 2856, 1613, 1506, 1244, $1088 \mathrm{~cm}^{-}$ 1. $m / z$ (ESI): $425\left(\mathrm{MNa}^{+}, 100 \%\right), 342(2 \%), 212(2 \%)$. HRMS Calcd. for $\mathrm{C}_{23} \mathrm{H}_{34} \mathrm{O}_{4} \mathrm{NaSi}$ $\left(\mathrm{MNa}^{+}\right)$: 425.2119. Found: 425.2117. HPLC analysis: $82 \%$ ee $(R, R$-Whelk-O1, 3\% $i$ PrOH in hexane, $1.0 \mathrm{ml} / \mathrm{min}$, $=254 \mathrm{~nm}, t_{\mathrm{R}}=19.8 \mathrm{~min}$, minor; $t_{\mathrm{R}}=25.5 \mathrm{~min}$, major). 
(2S,3R)-2-(4-Bromophenyl)-3-(tert-butyldimethylsilyloxy)-3-(4methoxyphenyl)propan-1-ol (16)

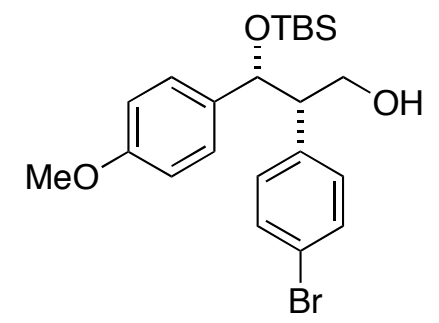

A solution of $\mathrm{Rh}_{2}(\mathrm{OOct})_{4}(3.9 \mathrm{mg}, 0.005 \mathrm{mmol}, 0.01$ eq.), compound 6a (126 mg, 0.5 mmol, 1 eq.) and 2,2-dimethylbutane ( $5 \mathrm{~mL})$ was heated under reflux for $45 \mathrm{~min}$, and then allowed to cool to room temperature. A solution of (S)-2-[2-(4-bromo-phenyl)-2diazo-acetoxy]-propionic acid ethyl ester (341 mg, $1.0 \mathrm{mmol}, 2$ eq.) in 2,2dimethylbutane $(20 \mathrm{~mL})$ was added via syringe pump at a rate of $6.8 \mathrm{ml} / \mathrm{h}$. After addition was complete, the reaction mixture was stirred for $1 \mathrm{~h}$, and then cooled to $0{ }^{\circ} \mathrm{C}$. Diisobutylaluminium hydride (1.0M in toluene, $3.0 \mathrm{ml}, 3.0 \mathrm{mmol}, 6 \mathrm{eq}$.) was added, and the reaction stirred for $2 \mathrm{~h}$ at $0{ }^{\circ} \mathrm{C}$ and then $10 \mathrm{~h}$ at room temperature. The reaction mixture was then poured into a 1:1 mix $(50 \mathrm{~mL})$ of $\mathrm{Et}_{2} \mathrm{O}$ and saturated sodium potassium tartrate solution, and the biphasic mixture stirred for $2 \mathrm{~h}$. The layers were separated, and the aqueous layer was extracted with 2 portions $\mathrm{Et}_{2} \mathrm{O}$. The combined organic fractions were dried $\left(\mathrm{Na}_{2} \mathrm{SO}_{4}\right)$ and concentrated in vacuo. Diastereoselectivity $(82 \%$ de) was determined by ${ }^{1} \mathrm{H}$ NMR of the crude reaction mixture, and the product purified by flash chromatography on silica gel using 10:1 hexane/ $\mathrm{Et}_{2} \mathrm{O}$ as eluant to isolate a mixture of the anti and syn isomers as a colorless oil, $56 \mathrm{mg}$; and the pure syn isomer as a colorless oil, $86 \mathrm{mg}$ (total yield: 63\%). [_] $]_{\mathrm{D}}+43.1$ (ee 82\%) (c 1.30, $\left.\mathrm{CHCl}_{3}\right) .{ }^{1} \mathrm{H}$ NMR $(500 \mathrm{MHz}$, $\left.\mathrm{CDCl}_{3}\right)$ : $7.36(\mathrm{~d}, 2 \mathrm{H}, J=8.5 \mathrm{~Hz}), 6.96(\mathrm{~d}, 2 \mathrm{H}, J=8.5 \mathrm{~Hz}), 6.94$ (d, 2H, $\left.J=8.5 \mathrm{~Hz}\right), 6.76$ (d, $2 \mathrm{H}, J=8.5 \mathrm{~Hz}$ ), 4.92 (d, 1H, J=7.0 Hz), 3.88 (dd, 1H, $J=11.0,7.0 \mathrm{~Hz}), 3.78$ (s, $3 \mathrm{H}), 3.75$ (dd, $1 \mathrm{H}, J=11.0,7.0 \mathrm{~Hz}$ ), 3.03 (dd, 1H, $J=12.5 \mathrm{~Hz}, 7.0 \mathrm{~Hz}$ ), 1.79 (br s, 1H), 0.82 (s, 9H), -0.07 (s, 3H), -0.27 (s, 3H). ${ }^{13} \mathrm{C}$ NMR (125 MHz, $\mathrm{CDCl}_{3}$ ):_158.7, 138.0, 134.2, 131.1, 130.9, 127.7, 120.6, 113.1, 76.1, 63.6, 56.0, 55.1, 25.7, 18.0, -4.7, -5.5. IR (film): 3402, 2927, 2856, 1613, 1513, 1488, 1245, 1112, $1009 \mathrm{~cm}^{-1} . \mathrm{m} / z$ (CI): $451\left(\mathrm{MH}^{+}, 5 \%\right), 345$ (76\%), 321 (98\%), 319 (100\%), 251 (93\%). HRMS Calcd. for $\mathrm{C}_{22} \mathrm{H}_{32} \mathrm{O}_{3} \mathrm{BrSi}\left(\mathrm{MH}^{+}\right)$: 451.1299. Found: 451.1295 . HPLC analysis: $82 \%$ ee $(R, R$-Whelk-O1, $3 \% i$-PrOH in hexane, $1.0 \mathrm{ml} / \mathrm{min},=254 \mathrm{~nm}, t_{\mathrm{R}}=13.1 \mathrm{~min}$, minor; $t_{\mathrm{R}}=17.6 \mathrm{~min}$, major). 
(2S,3R)-3-(tert-Butyldimethylsilyloxy)-3-(4-methoxyphenyl)-2-(4(trifluoromethyl)phenyl)propan-1-ol (17)

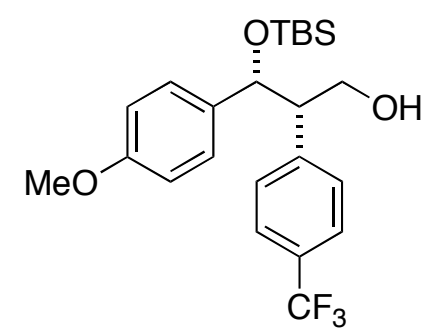

A solution of $\mathrm{Rh}_{2}(\mathrm{OOct})_{4}(3.9 \mathrm{mg}, 0.005 \mathrm{mmol}, 0.01 \mathrm{eq}$ ), compound $6 \mathrm{a}(126 \mathrm{mg}, 0.5$ mmol, 1 eq.) and 2,2-dimethylbutane ( $5 \mathrm{~mL}$ ) was heated under reflux for $45 \mathrm{~min}$, and then allowed to cool to room temperature. A solution of 2-[2-diazo-2-(4-trifluoromethylphenyl)-acetoxy]-propionic acid ethyl ester $(330 \mathrm{mg}, 1.0 \mathrm{mmol}, 2$ eq.) in 2,2dimethylbutane $(10 \mathrm{~mL})$ was added via syringe pump at a rate of $3.33 \mathrm{ml} / \mathrm{h}$. After addition was complete, the reaction mixture was stirred for $1 \mathrm{~h}$, and then cooled to $0{ }^{\circ} \mathrm{C}$. Diisobutylaluminium hydride (1.0M in toluene, $3.0 \mathrm{ml}, 3.0 \mathrm{mmol}, 6$ eq.) was added, and the reaction stirred for $2 \mathrm{~h}$ at $0{ }^{\circ} \mathrm{C}$ and then $10 \mathrm{~h}$ at room temperature. The reaction mixture was then poured into a 1:1 mix $(50 \mathrm{~mL})$ of $\mathrm{Et}_{2} \mathrm{O}$ and saturated sodium potassium tartrate solution, and the biphasic mixture stirred for $2 \mathrm{~h}$. The layers were separated, and the aqueous layer was extracted with 2 portions $\mathrm{Et}_{2} \mathrm{O}$. The combined organic fractions were dried $\left(\mathrm{Na}_{2} \mathrm{SO}_{4}\right)$ and concentrated in vacuo. Diastereoselectivity $(80 \%$ de) was determined by ${ }^{1} \mathrm{H}$ NMR of the crude reaction mixture, and the product purified by flash chromatography on silica gel using 10:1 hexane/ $\mathrm{Et}_{2} \mathrm{O}$ as eluant to isolate a the anti isomer as a colorless oil, $12 \mathrm{mg}$; and the pure syn isomer as a colorless oil, $131 \mathrm{mg}$ (total yield: 65\%). [_]D +47.0 (ee 76\%) (c 1.23, $\left.\mathrm{CHCl}_{3}\right) .{ }^{1} \mathrm{H} \mathrm{NMR}\left(500 \mathrm{MHz}, \mathrm{CDCl}_{3}\right)$ : _7.51 (d, 2H, $J=8.5 \mathrm{~Hz}), 7.21$ (d, 2H, $J=8.5 \mathrm{~Hz}), 6.99$ (d, 2H, $J=8.5 \mathrm{~Hz}), 6.78(\mathrm{~d}, 2 \mathrm{H}, J=8.5 \mathrm{~Hz}), 4.95$ (d, $1 \mathrm{H}, J=6.0 \mathrm{~Hz}$ ), 3.91 (dd, 1H, $J=11.5,7.0 \mathrm{~Hz}$ ), 3.82-3.75 (m, 1H), 3.79 (s, 3H), 3.14 (dd, 1H, $J=13.0,6.0 \mathrm{~Hz}$ ), 1.83 (br s, 1H), 0.79 (s, 9H), -0.10 (s, 3H), -0.29 (s, 3H). ${ }^{13} \mathrm{C}$ NMR (75 MHz, $\mathrm{CDCl}_{3}$ ):_158.9, 143.6, 134.3, 129.7, 129.0 (q, $\left.J=32.5 \mathrm{~Hz}\right), 127.7,124.7$ (q, $J=4.0 \mathrm{~Hz}$ ), 124.3 (q, $J=271.5 \mathrm{~Hz}$ ), 113.2, 76.0, 63.6, 56.4, 55.1, 25.6, 17.9, -4.8, -5.6. IR (film): 3382, 2952, 1614, 1586, 1514, 1167, $836 \mathrm{~cm}^{-1} . \mathrm{m} / \mathrm{z}$ (EI): 463 ( $\left.\mathrm{MNa}^{+}, 3 \%\right), 425$ (5\%), 383 (10\%), 278 (11\%), 251 (100\%). Anal. calcd. for $\mathrm{C}_{23} \mathrm{H}_{31} \mathrm{O}_{3} \mathrm{~F}_{3} \mathrm{Si}: \mathrm{C}, 62.70 ; \mathrm{H}$, 7.09. Found: $\mathrm{C}, 63.08 ; \mathrm{H}, 7.21$. HPLC analysis: $76 \%$ ee $(R, R$-Whelk-O1, $3 \% i-\mathrm{PrOH}$ in hexane, $1.0 \mathrm{ml} / \mathrm{min},=254 \mathrm{~nm}, t_{\mathrm{R}}=9.1 \mathrm{~min}$, minor; $t_{\mathrm{R}}=11.6 \mathrm{~min}$, major). 
(2R,3R)-Methyl 2-(4-bromophenyl)-3-(tert-butyldimethylsilyloxy)-3-(4methoxyphenyl)propanoate (21)

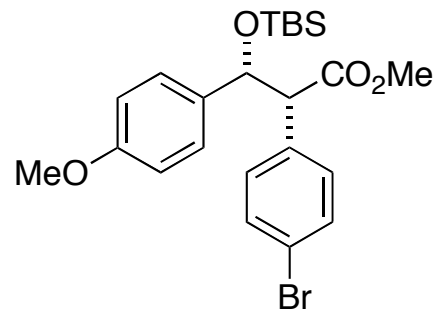

A solution of methyl phenyldiazoacetate 7 (1.55 g, $6.06 \mathrm{mmol}, 1.50$ eq.) in 2,2dimethylbutane $(40 \mathrm{~mL})$ was added to a solution of $\mathrm{Rh}_{2}(S-\mathrm{PTTL})_{4}(50 \mathrm{mg}, 0.04 \mathrm{mmol}$, 0.01 eq.) and compound $6 \mathbf{6}$ (1.02 g, $4.04 \mathrm{mmol}, 1.00$ eq.) and 2,2-dimethylbutane (35 $\mathrm{mL}$ ) under reflux via syringe pump at a rate of $2 \mathrm{ml} / \mathrm{h}$. After addition was complete, the reaction mixture was stirred at $50{ }^{\circ} \mathrm{C}$ for $30 \mathrm{~min}$, and then allowed to cool to room temperature and concentrated in vacuo. Diastereoselectivity ( $83 \%$ de) was determined by ${ }^{1} \mathrm{H}$ NMR of the crude reaction mixture, and the products purified by flash chromatography on silica gel using $18: 1$ hexane/ $/ \mathrm{Et}_{2} \mathrm{O}$ as eluant to provide a mixture of anti and syn isomers as a colorless oil, $244 \mathrm{mg}$; and pure syn isomer as a colorless oil, $1.67 \mathrm{~g}$ (total yield: $86 \%)$. $[\alpha]_{\mathrm{D}}+72.2(97 \% \mathrm{ee})\left(c\right.$ 1.31, $\left.\mathrm{CHCl}_{3}\right) .{ }^{1} \mathrm{H}$ NMR $(400 \mathrm{MHz}$, $\mathrm{CDCl}_{3}$ ): 87.44 (d, 2H, J=8.5 Hz), 7.29 (d, 2H, J=8.5 Hz), 7.20 (d, 2H, J=8.5 Hz), 6.81 (d, $2 \mathrm{H}, J=8.5 \mathrm{~Hz}$ ), 5.08 (d, $1 \mathrm{H}, J=8.0 \mathrm{~Hz}), 3.80$ (s, 3H), 3.73 (d, $1 \mathrm{H}, J=8.0 \mathrm{~Hz}), 3.48$ (s, $3 \mathrm{H})$, 0.66 (s, 9H), -0.30 (s, 3H), -0.37 (s, 3H). ${ }^{13} \mathrm{C}$ NMR $\left(75 \mathrm{MHz}, \mathrm{CDCl}_{3}\right): \delta 171.8,159.0$, 135.4, 134.5, 131.3, 131.1, 128.0, 121.3, 113.3, 76.5, 61.0, 55.1, 51.8, 25.5, 17.8, -4.9, 5.7. IR $\left(\mathrm{CHCl}_{3}\right): 2953.2929,2895,2856,1736,1612,1512,1250,1082,837 \mathrm{~cm}^{-1} . \mathrm{m} / \mathrm{z}$ (EI): $479\left(\mathrm{M}^{+}, 10 \%\right), 477$ (9\%), 251 (100\%). Anal Calcd. For $\mathrm{C}_{23} \mathrm{H}_{31} \mathrm{O}_{4} \mathrm{SiBr}$ : C, 57.61; H, 6.52. Found: $\mathrm{C}, 57.80 ; \mathrm{H}, 6.50$. . HPLC analysis determined by conversion of the product 21 to the alcohol 16: $97 \%$ ee $(R, R$-Whelk-O1, $3 \% i-\mathrm{PrOH}$ in hexane, $1.0 \mathrm{ml} / \mathrm{min},=254$ $\mathrm{nm}, t_{\mathrm{R}}=13.1 \mathrm{~min}$, minor; $t_{\mathrm{R}}=17.6$ min, major). 
(2R,3R)-Methyl 3-(tert-butyldimethylsilyloxy)-2,3-bis(4-methoxyphenyl)propanoate (22)

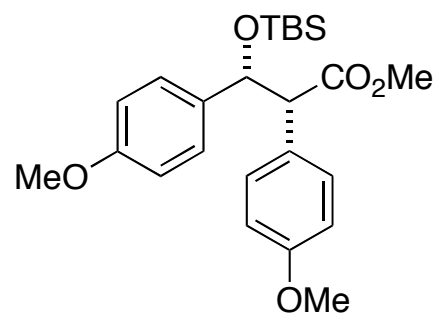

A solution of methyl phenyldiazoacetate 7 (225 mg, $1.09 \mathrm{mmol}, 2.00$ eq.) in 2,2dimethylbutane $(6 \mathrm{~mL})$ was added to a solution of $\mathrm{Rh}_{2}(S \text {-PTTL })_{4}(7 \mathrm{mg}, 0.006 \mathrm{mmol}$, 0.01 eq.) and compound $6 \mathbf{6}$ (138 $\mathrm{mg}, 0.55 \mathrm{mmol}, 1.00$ eq.) and 2,2-dimethylbutane (3 $\mathrm{mL}$ ) under reflux via syringe pump at a rate of $2 \mathrm{ml} / \mathrm{h}$. After addition was complete, the reaction mixture was stirred at $50{ }^{\circ} \mathrm{C}$ for $30 \mathrm{~min}$, and then allowed to cool to room temperature and concentrated in vacuo. Diastereoselectivity ( $94 \%$ de) was determined by ${ }^{1} \mathrm{H}$ NMR of the crude reaction mixture, and the products purified by flash chromatography on silica gel using $18: 1$ hexane/ $\mathrm{Et}_{2} \mathrm{O}$ as eluant to provide the anti isomer as a pale yellow oil, $5 \mathrm{mg}$; a mixture of anti and syn isomer as a pale yellow oil, $64 \mathrm{mg}$; and pure syn isomer as a pale yellow oil, $120 \mathrm{mg}$ (total yield: $88 \%)$. $[\alpha]_{\mathrm{D}}+75.2(96 \% \mathrm{ee})$ (c 1.45, $\left.\mathrm{CHCl}_{3}\right) .{ }^{1} \mathrm{H}$ NMR (500 MHz, $\left.\mathrm{CDCl}_{3}\right): \delta 7.33$ (d, 2H, J=8.5 Hz), $7.23(\mathrm{~d}, 2 \mathrm{H}$, $J=9.0 \mathrm{~Hz}), 6.85(\mathrm{~d}, 2 \mathrm{H}, J=9.0 \mathrm{~Hz}), 6.81(\mathrm{~d}, 2 \mathrm{H}, J=8.5 \mathrm{~Hz}), 5.05(\mathrm{~d}, 1 \mathrm{H}, J=9.0 \mathrm{~Hz}), 3.81$ (s, 3H), 3.80 (s, 3H), 3.72 (d, 1H, J=9.0 Hz), 3.45 (s, 3H), 0.65 (s, 9H), -0.33 (s, 3H), 0.37 (s, $3 \mathrm{H}) .{ }^{13} \mathrm{C}$ NMR $\left(125 \mathrm{MHz}, \mathrm{CDCl}_{3}\right): \delta 172.4,158.9,158.8,135.0,130.4,128.6$, 128.0, 113.4, 113.1, 76.6, 60.7, 55.2, 55.0, 51.5, 25.4, 17.8, -5.0, -5.7. IR $\left(\mathrm{CHCl}_{3}\right): 2999$, 2953, 2929, 2856, 1734, 1612, 1512, 1248, 1084, 854, $777 \mathrm{~cm}^{-1} . \mathrm{m} / z$ (ESI): $453\left(\mathrm{MNa}^{+}\right.$, 19\%), 299 (86\%). Found: 453.2087. Anal Calcd. For $\mathrm{C}_{24} \mathrm{H}_{34} \mathrm{O}_{5} \mathrm{Si}$ : C, 66.94; H, 7.96. Found: C, 66.94; H, 8.23. HPLC analysis: 96\%ee (Chiralcel OD-H, $1 \% i$-PrOH in hexane, $1.0 \mathrm{ml} / \mathrm{min},{ }_{-}=254 \mathrm{~nm}, t_{\mathrm{R}}=5.2 \mathrm{~min}$, minor; $t_{\mathrm{R}}=6.7 \mathrm{~min}$, major). 


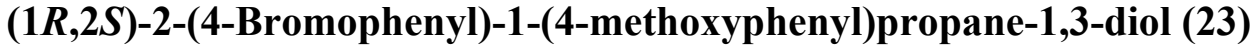

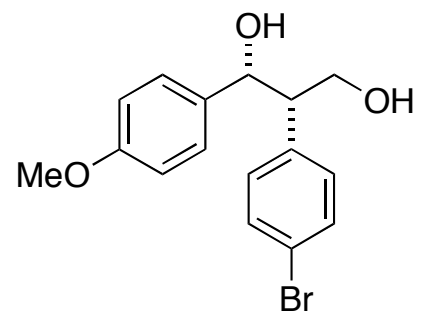

A solution of compound 16 (61 mg, $0.13 \mathrm{mmol}, 1.0$ eq.; 97\%ee derived from reduction of compound 21 with DIBAL) in THF (5 mL) was cooled to $0{ }^{\circ} \mathrm{C}$. Tetrabutylammonium fluoride (1.0M in THF, $0.15 \mathrm{~mL}, 0.15 \mathrm{mmol}, 1.1$ eq.) was added, and the reaction mixture was stirred for $1 \mathrm{~h}$, by which time TLC indicated that the starting material was consumed. $\mathrm{CH}_{2} \mathrm{Cl}_{2}$ was added, and the reaction mixture was washed with brine, dried $\left(\mathrm{MgSO}_{4}\right)$ and concentrated in vacuo. The residue was purified by flash chromatography on silica gel using 4:1 $\mathrm{Et}_{2} \mathrm{O} /$ hexane as eluant to isolate a white solid, $40 \mathrm{mg}(86 \%)$. Recrystallized from hexane/ $\mathrm{Et}_{2} \mathrm{O}$ to give crystals suitable for x-ray analysis. Mp 123-125 ${ }^{\circ} \mathrm{C}$. [_] $]_{\mathrm{D}}+75.3$ $\left(c\right.$ 0.98, $\left.\mathrm{CHCl}_{3}\right) .{ }^{1} \mathrm{H}$ NMR $\left(500 \mathrm{MHz}, \mathrm{CDCl}_{3}\right): \_7.46(\mathrm{~d}, 2 \mathrm{H}, J=8.5 \mathrm{~Hz}), 7.17$ (d, 2H, J=9.0 Hz), $7.11(\mathrm{~d}, 2 \mathrm{H}, J=8.5 \mathrm{~Hz}), 6.86$ (d, 2H, J=9.0 Hz), 5.02 (dd, 1H, J=6.5, 3.0 Hz), 3.86$3.74(\mathrm{~m}, 2 \mathrm{H}), 3.81(\mathrm{~s}, 3 \mathrm{H}), 3.12(\mathrm{dd}, 1 \mathrm{H}, J=12.5,6.5 \mathrm{~Hz}), 2.18(\mathrm{~s}, 1 \mathrm{H}), 2.03(\mathrm{~d}, 1 \mathrm{H}, J=3.0$ $\mathrm{Hz}) .{ }^{13} \mathrm{C} \mathrm{NMR}\left(75 \mathrm{MHz}, \mathrm{CDCl}_{3}\right)$; _ 159.3, 137.9, 133.9, 131.6, 130.9, 127.7, 121.2, 113.8, 75.2, 64.2, 55.2, 55.0. IR $\left(\mathrm{CH}_{2} \mathrm{Cl}_{2}\right): 3421,2904,2850,1724,1612,1512,1250$, 1153, 1034, 906, 833, $729 \mathrm{~cm}^{-1} . \mathrm{m} / z$ (ESI): $361\left(\mathrm{MNa}^{+}\right.$with $\left.{ }^{81} \mathrm{Br}, 65 \%\right), 359\left(\mathrm{MNa}^{+}\right.$with $\left.{ }^{79} \mathrm{Br}, 64 \%\right), 200$ (49\%), 102 (100\%). Anal. Calcd. For $\mathrm{C}_{16} \mathrm{H}_{17} \mathrm{BrO}_{3}$ : C, 56.99; H, 5.08. Found: C, 57.19; H, 5.19.

(2S,3S,E)-3-(tert-Butyldimethylsilyloxy)-2,5-diphenylpent-4-en-1-ol (25)<smiles>OC[C@H](c1ccccc1)[C@H]([OH2+])/C=C/c1ccccc1</smiles>

A solution of $\mathrm{Rh}_{2}(\mathrm{OOct})_{4}(7.8 \mathrm{mg}, 0.01 \mathrm{mmol}, 0.02$ eq.), compound 24 (124 mg, 0.5 mmol, 1 eq.) and 2,2-dimethylbutane $(5 \mathrm{~mL})$ was heated under reflux for $45 \mathrm{~min}$, and then allowed to cool to room temperature. A solution of compound 11 (262 $\mathrm{mg}, 1.0$ mmol, 2 eq.) in 2,2-dimethylbutane $(10 \mathrm{~mL})$ was added via syringe pump at a rate of 3.33 $\mathrm{ml} / \mathrm{h}$. After addition was complete, the reaction mixture was stirred for $1 \mathrm{~h}$, and then 
cooled to $0{ }^{\circ} \mathrm{C}$. Diisobutylaluminium hydride (1.0M in toluene, $3.0 \mathrm{ml}, 3.0 \mathrm{mmol}, 6$ eq.) was added, and the reaction stirred for $2 \mathrm{~h}$ at $0{ }^{\circ} \mathrm{C}$ and then $10 \mathrm{~h}$ at room temperature. The reaction mixture was then poured into a 1:1 mix $(50 \mathrm{~mL})$ of $\mathrm{Et}_{2} \mathrm{O}$ and saturated sodium potassium tartrate solution, and the biphasic mixture stirred for $2 \mathrm{~h}$. The layers were separated, and the aqueous layer was extracted with 2 portions $\mathrm{Et}_{2} \mathrm{O}$. The combined organic fractions were dried $\left(\mathrm{Na}_{2} \mathrm{SO}_{4}\right)$ and concentrated in vacuo. Purified by flash chromatography on silica gel using 10:1 hexane/ $\mathrm{Et}_{2} \mathrm{O}$ as eluant to isolate a colorless oil, $93 \mathrm{mg}(51 \%)$. [_]D +2.96 (ee 80\%) (c 1.08, $\left.\mathrm{CHCl}_{3}\right) .{ }^{1} \mathrm{H}$ NMR $\left(500 \mathrm{MHz}, \mathrm{CDCl}_{3}\right)$ :_7.35$7.31(\mathrm{~m}, 5 \mathrm{H}), 7.28-7.22(\mathrm{~m}, 5 \mathrm{H}), 6.47$ (d, 1H, $J=16.0 \mathrm{~Hz}), 6.09$ (dd, 1H, $J=16.0,7.0 \mathrm{~Hz})$, 4.63 (t, $1 \mathrm{H}, J=5.5 \mathrm{~Hz}), 4.31-4.23$ (m, 1H), 3.96-3.76 (m, 1H), 3.15 (dd, $1 \mathrm{H}, J=12.0,6.0$ Hz), 2.23 (t, 1H, J=5.5 Hz), 0.90 (s, 9H), 0.06 (s, 3H), 0.03 (s, 3H). ${ }^{13} \mathrm{C}$ NMR (75 MHz, $\mathrm{CDCl}_{3}$ ): $\delta 139.0,136.8,131.0,130.0,129.1,128.5,128.3,127.6,126.9,126.4,76.2,63.8$, 54.7, 25.8, 18.1, -4.3, -5.2. IR ( $\left.\mathrm{CHCl}_{3}\right): 3388,3028,2955,2928,2856,1495,1254,1086$, 835, $777 \mathrm{~cm}^{-1} . \mathrm{m} / z$ (ESI): $391\left(\mathrm{MNa}^{+}, 100 \%\right), 219(10 \%)$. HRMS (ESI) calcd. For $\mathrm{C}_{23} \mathrm{H}_{32} \mathrm{O}_{2} \mathrm{NaSi}\left(\mathrm{MNa}^{+}\right)$: 391.2064. Found: 391.2065. HPLC analysis: $80 \%$ ee (Chiralcel OD-H, $1 \% i-\mathrm{PrOH}$ in hexane, $1.0 \mathrm{ml} / \mathrm{min}, \quad=254 \mathrm{~nm}, t_{\mathrm{R}}=17.3 \mathrm{~min}$, minor; $t_{\mathrm{R}}=18.9 \mathrm{~min}$, major). 


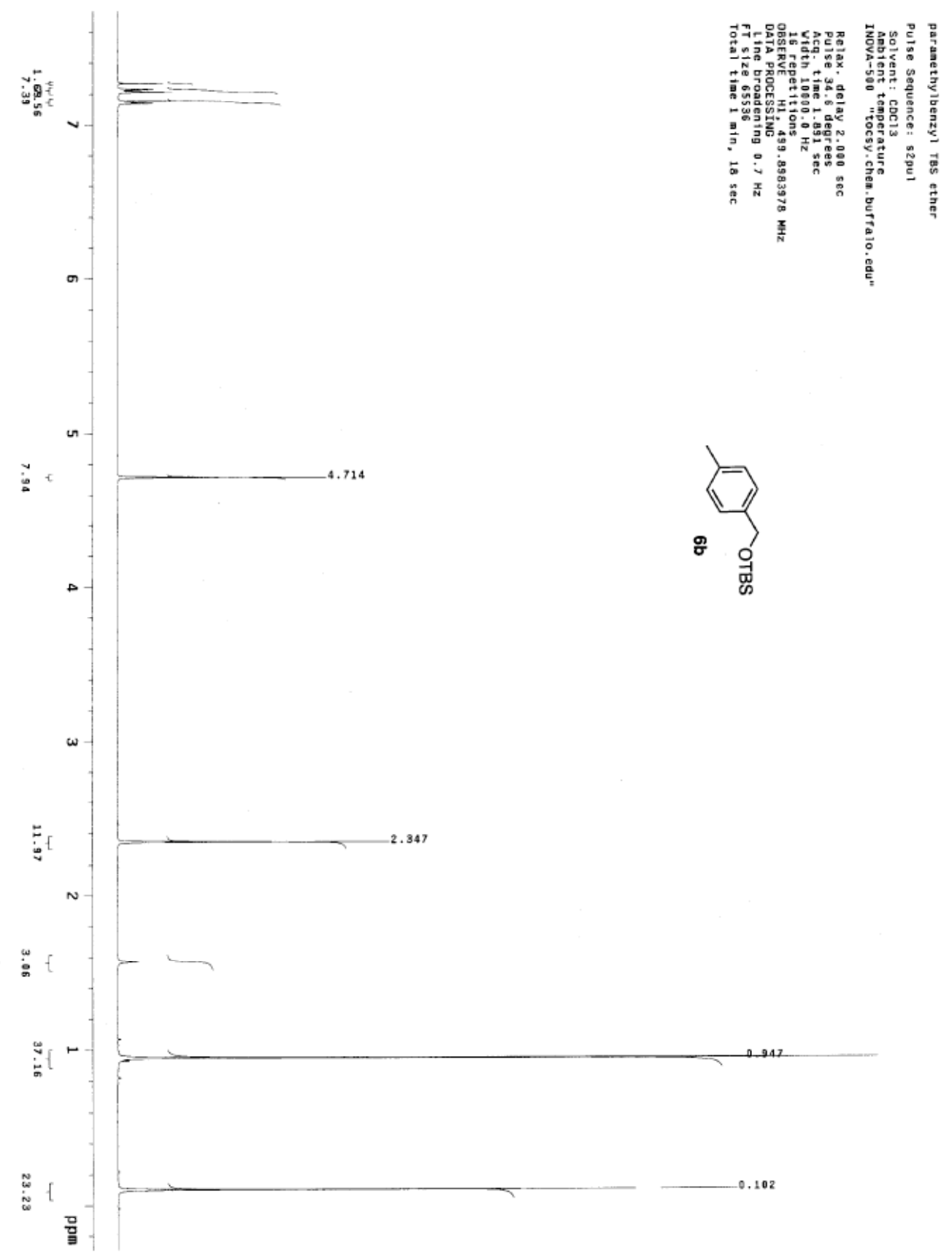




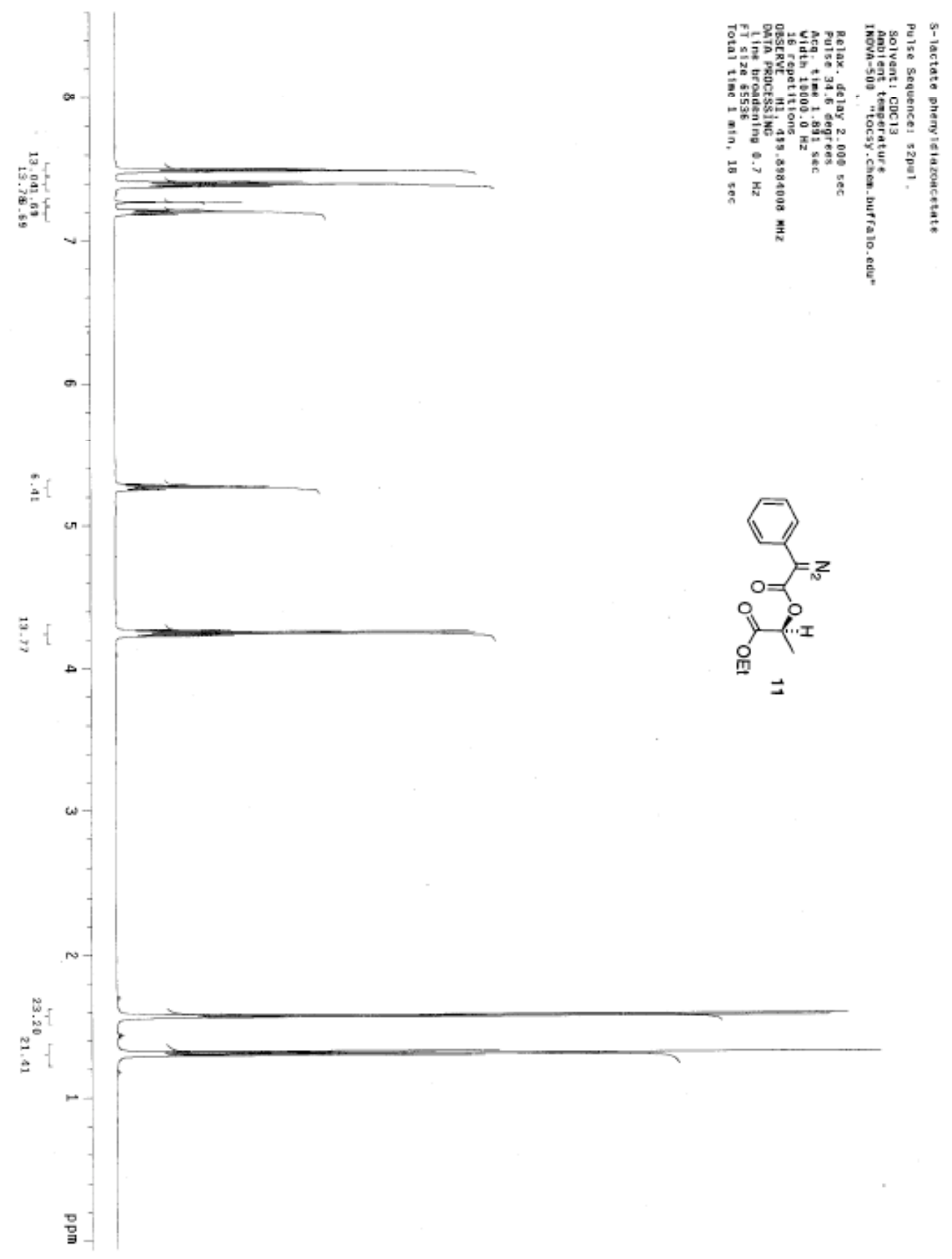




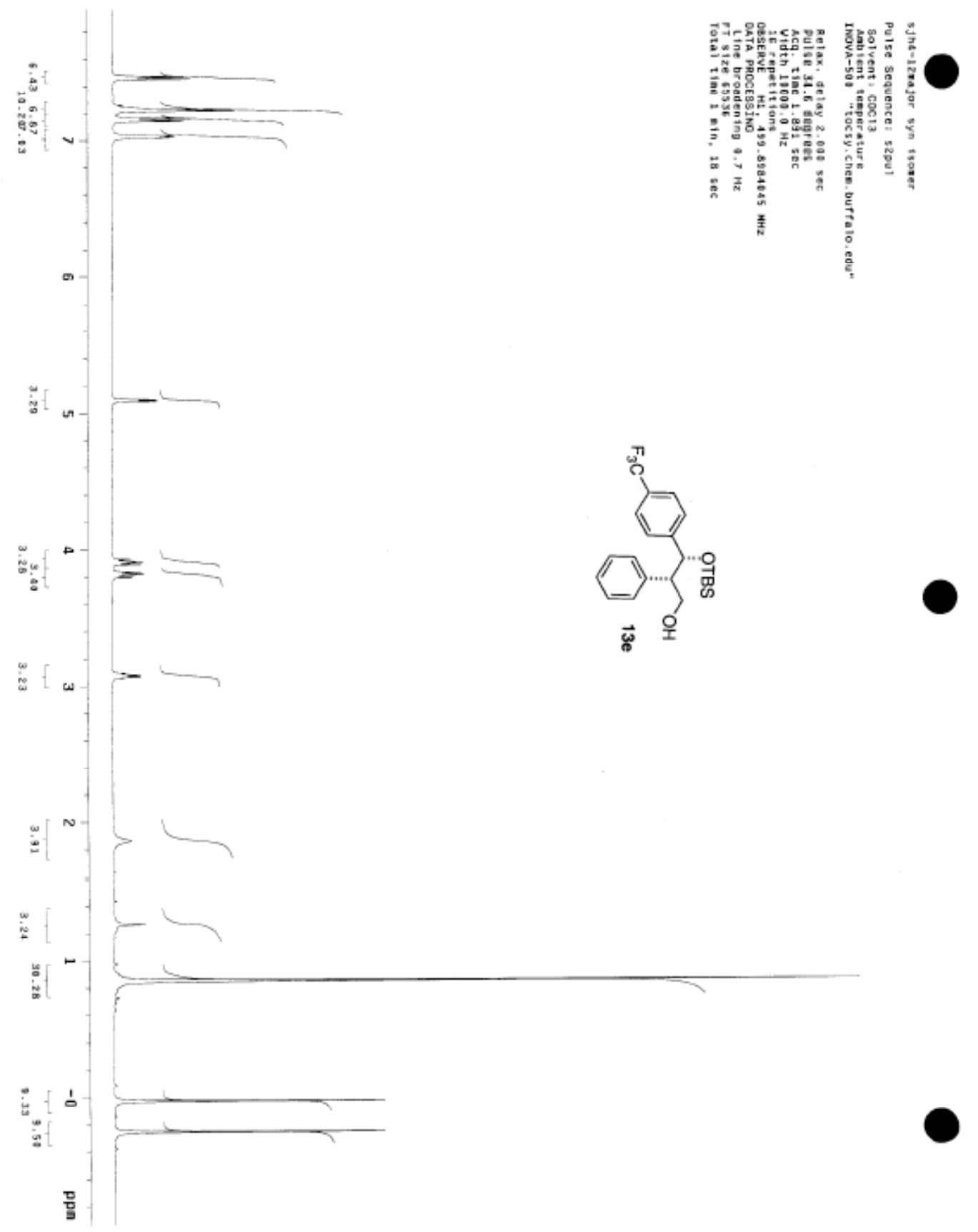

S-36 


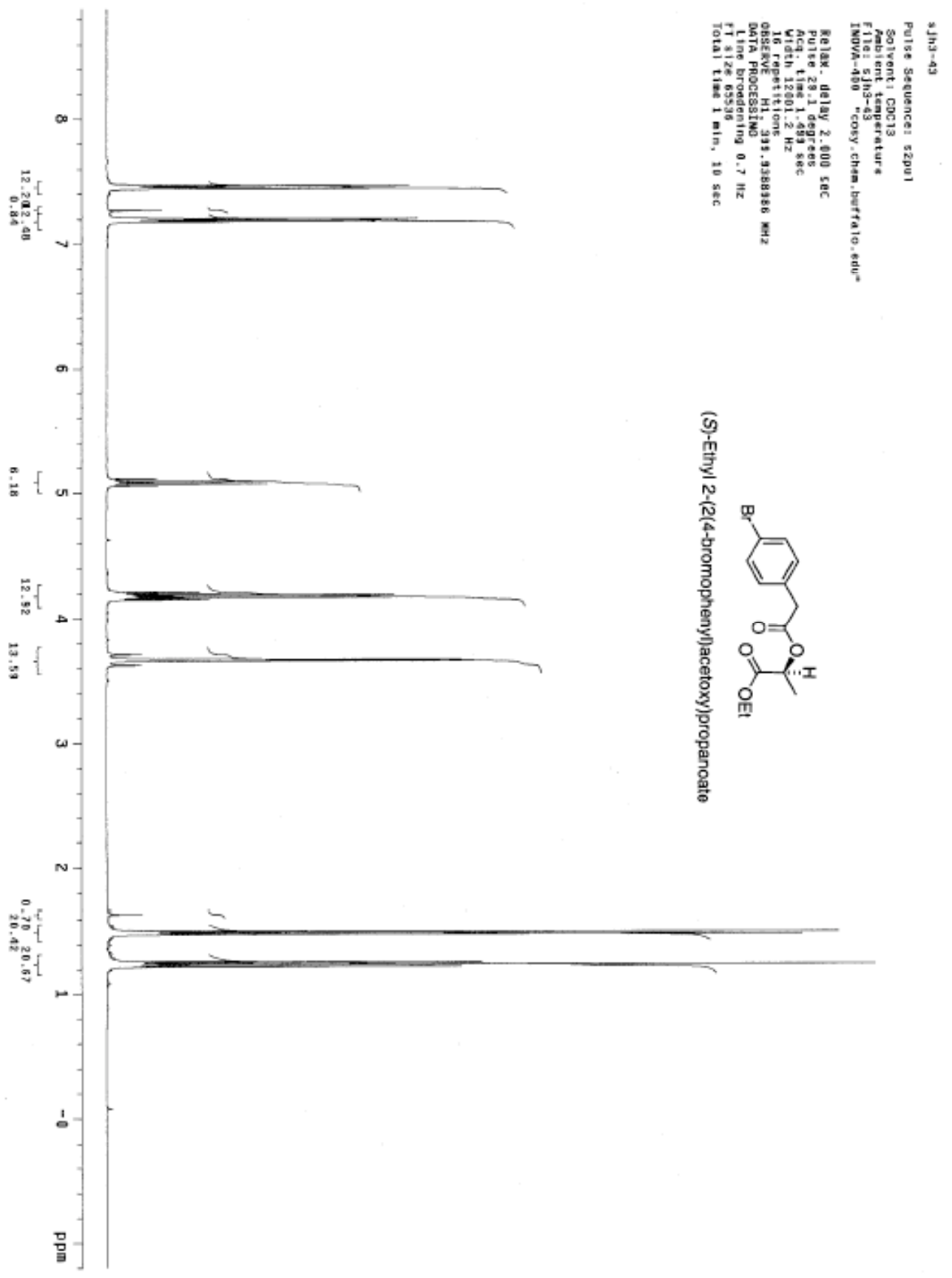




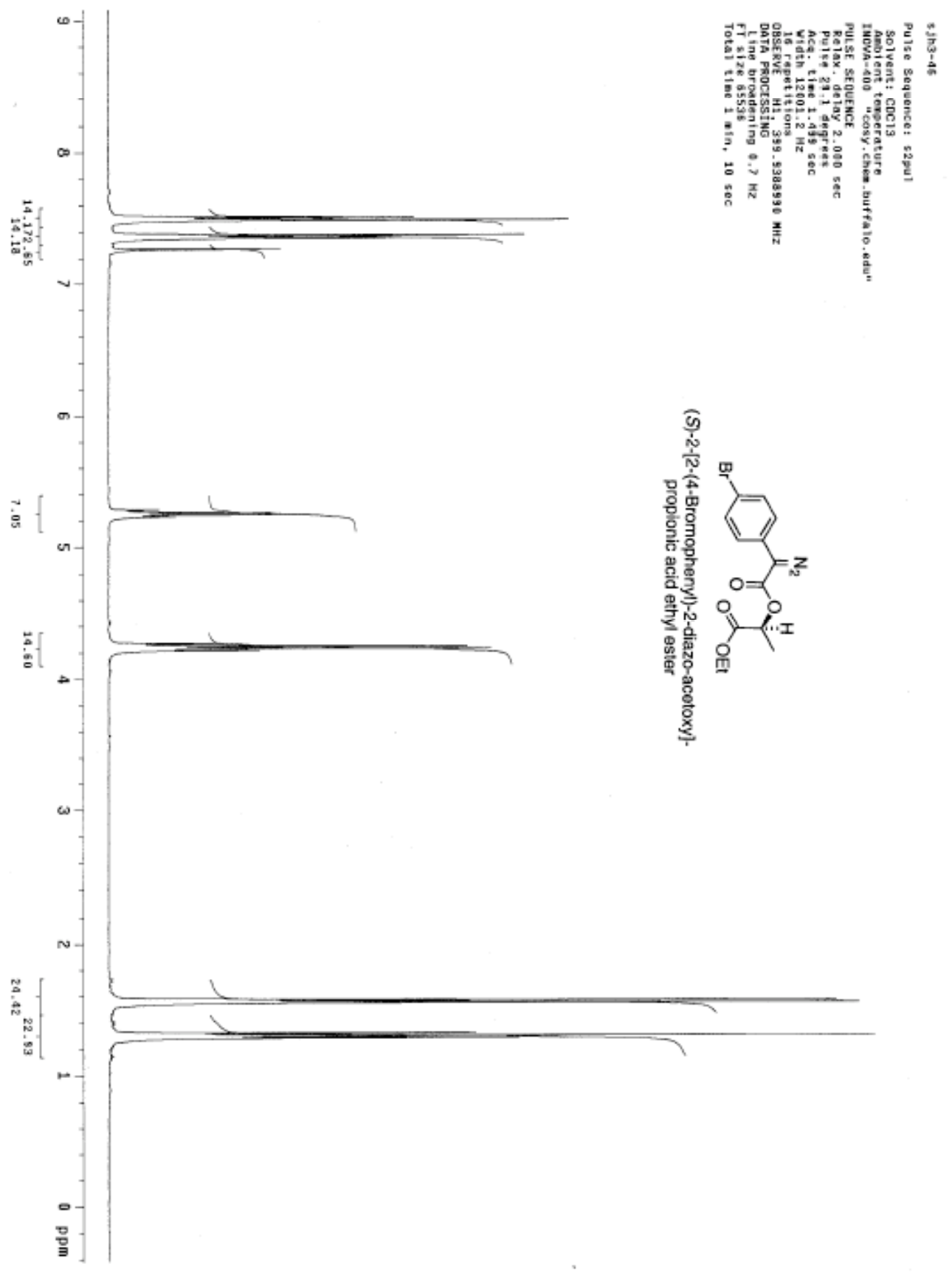




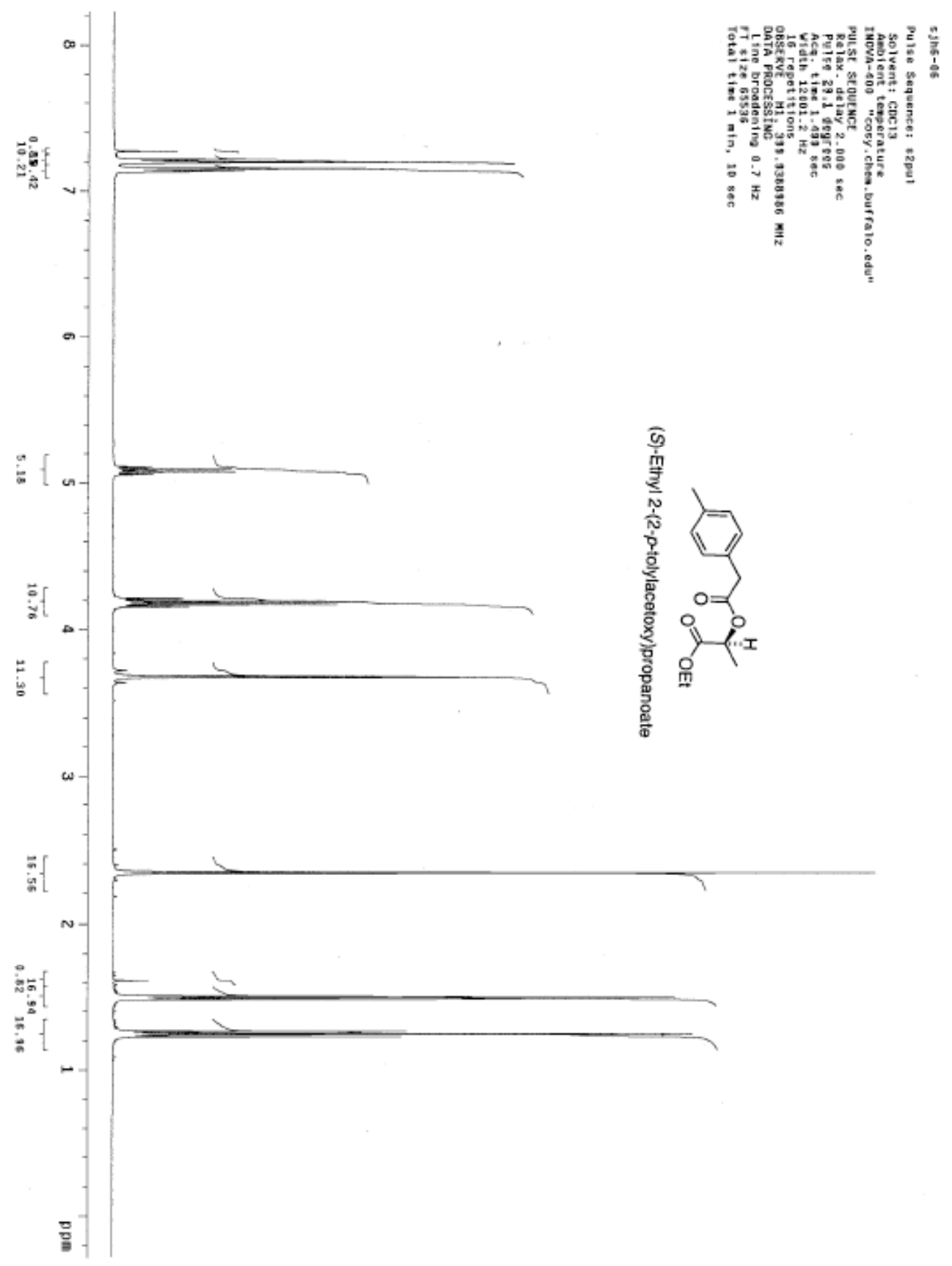




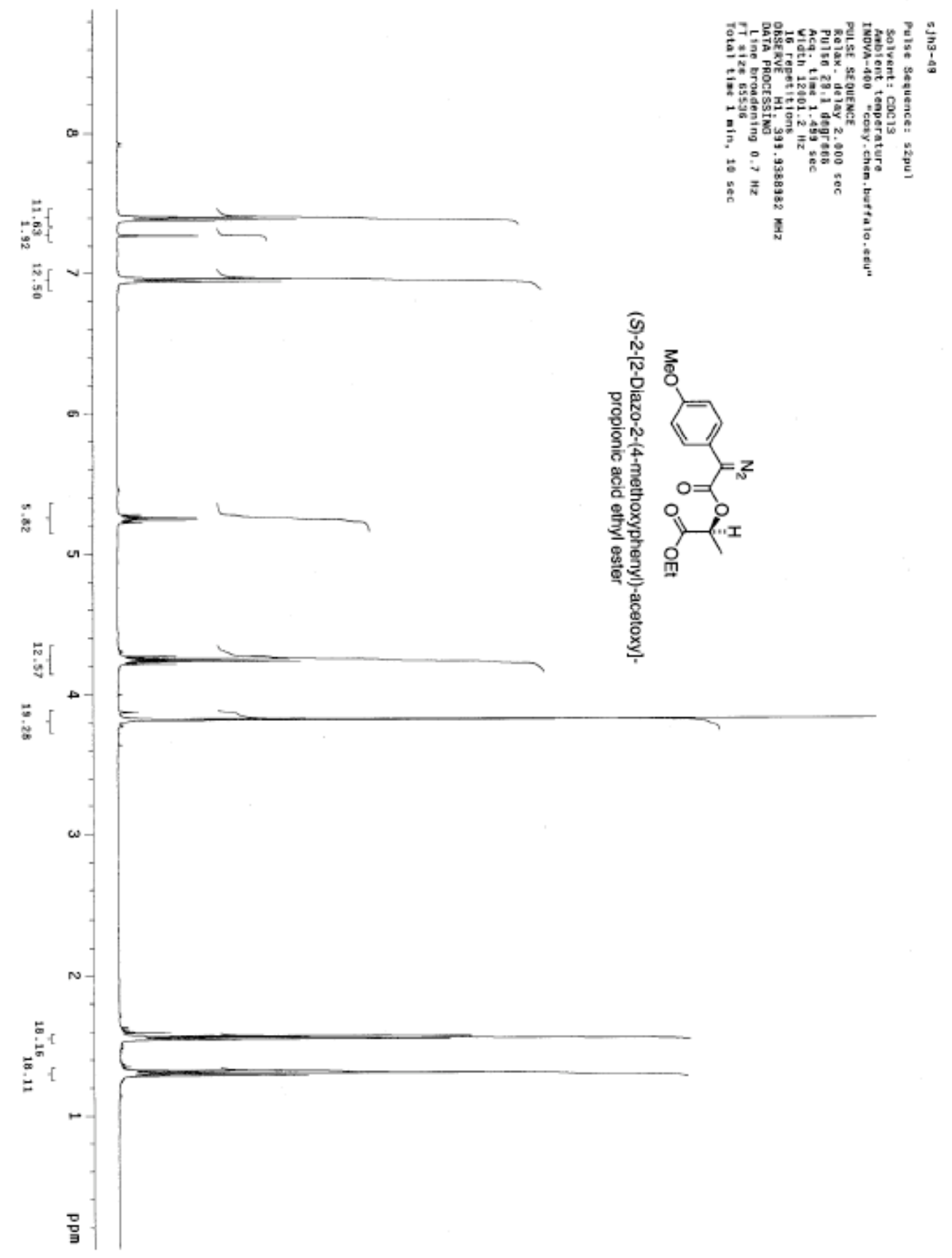




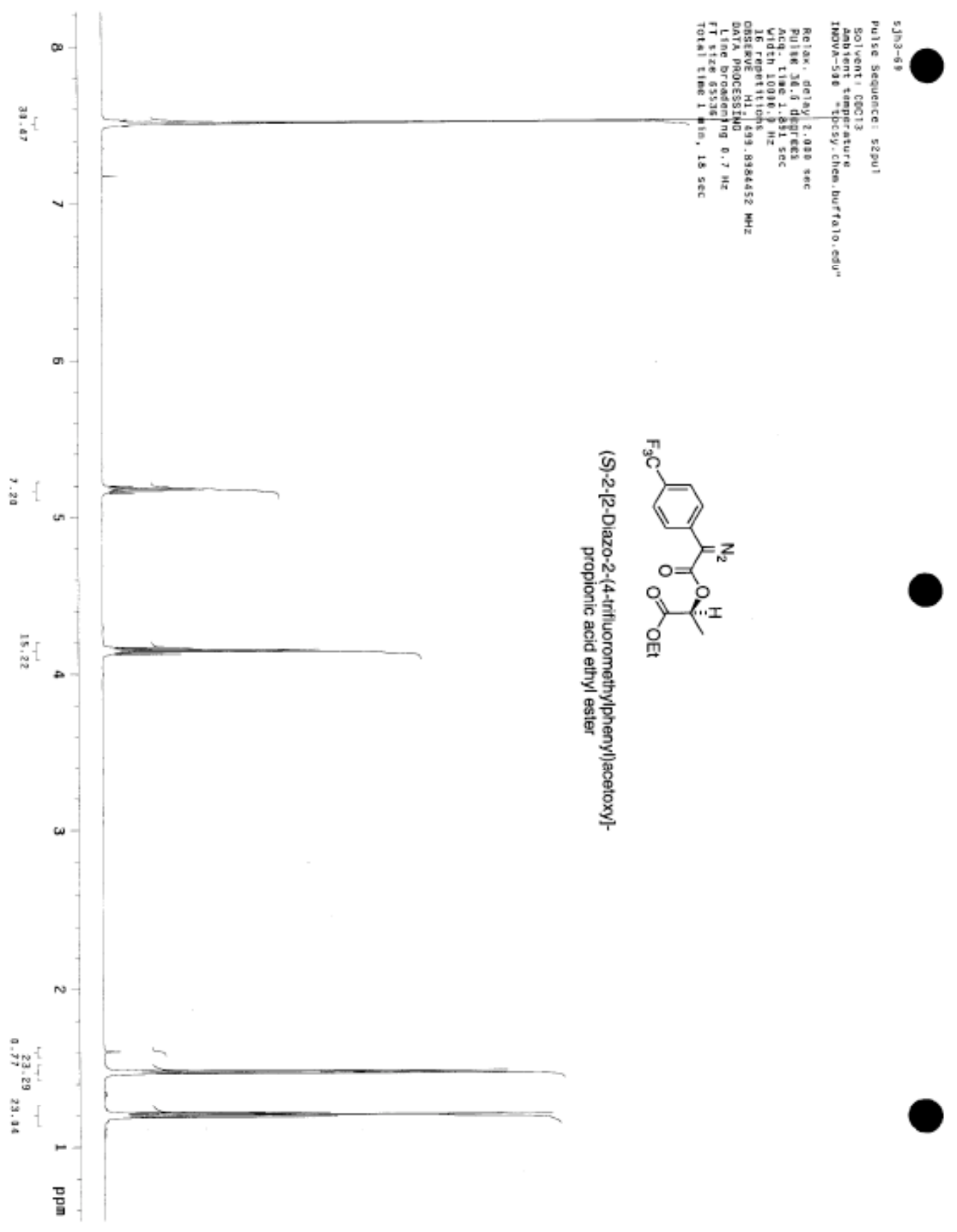




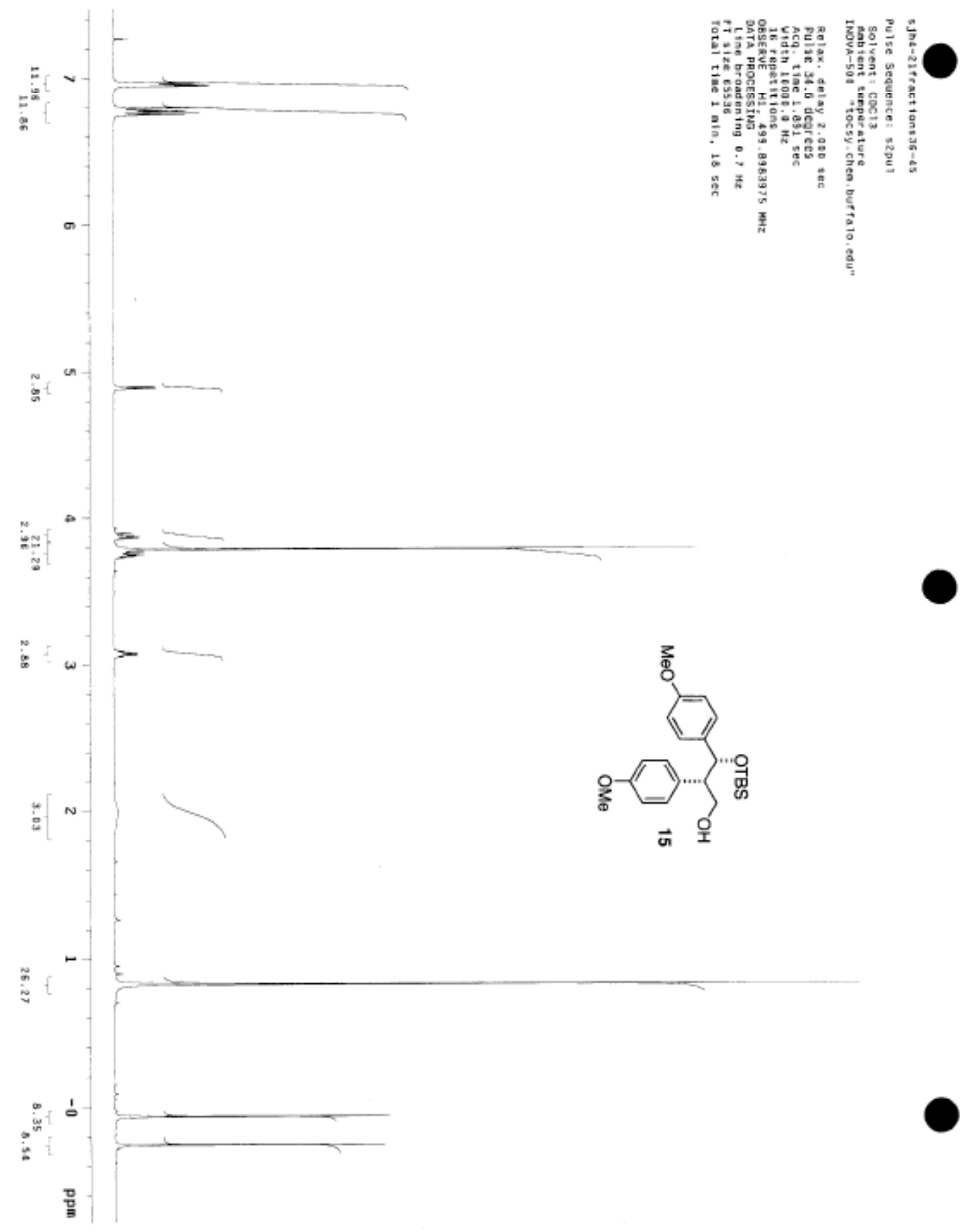




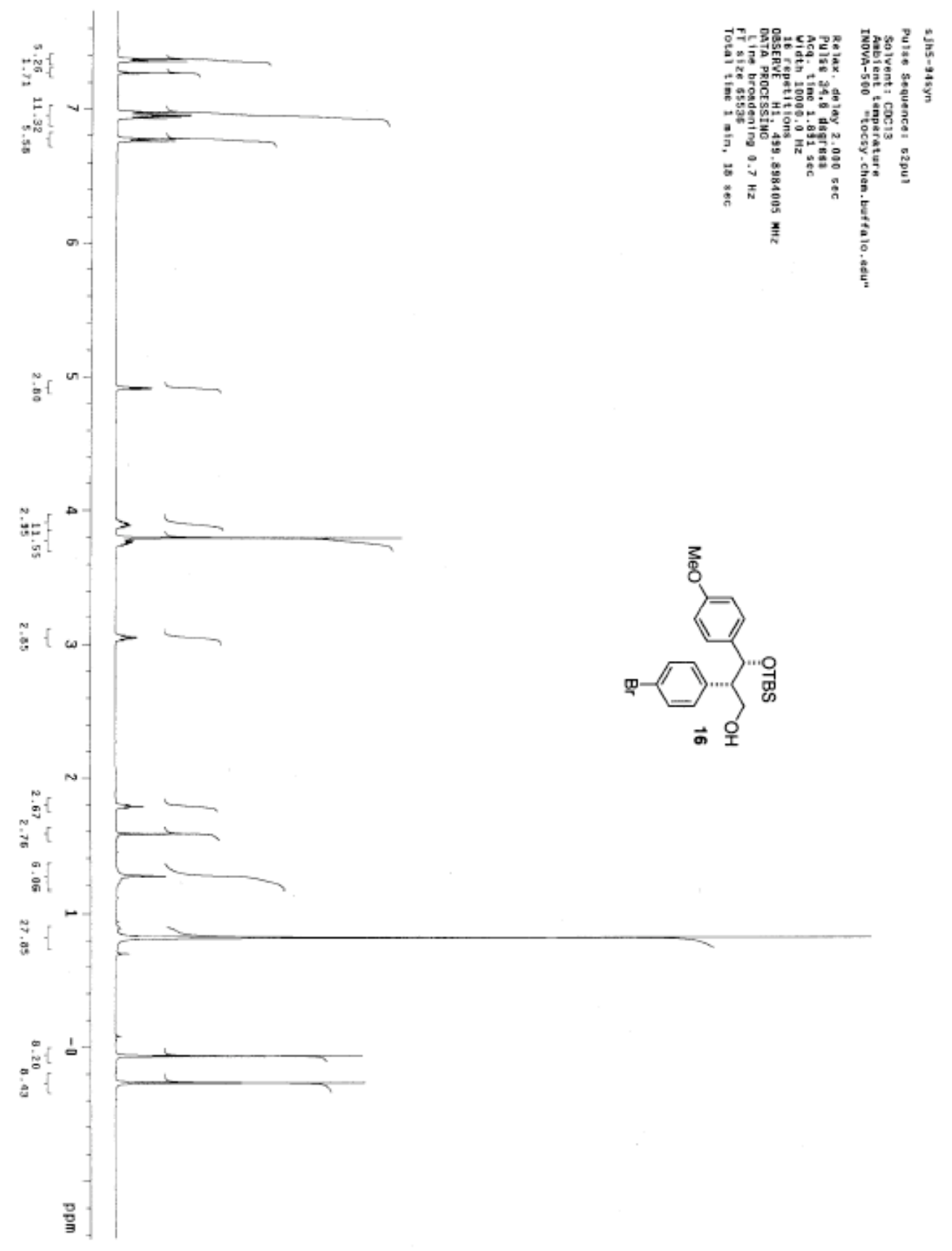




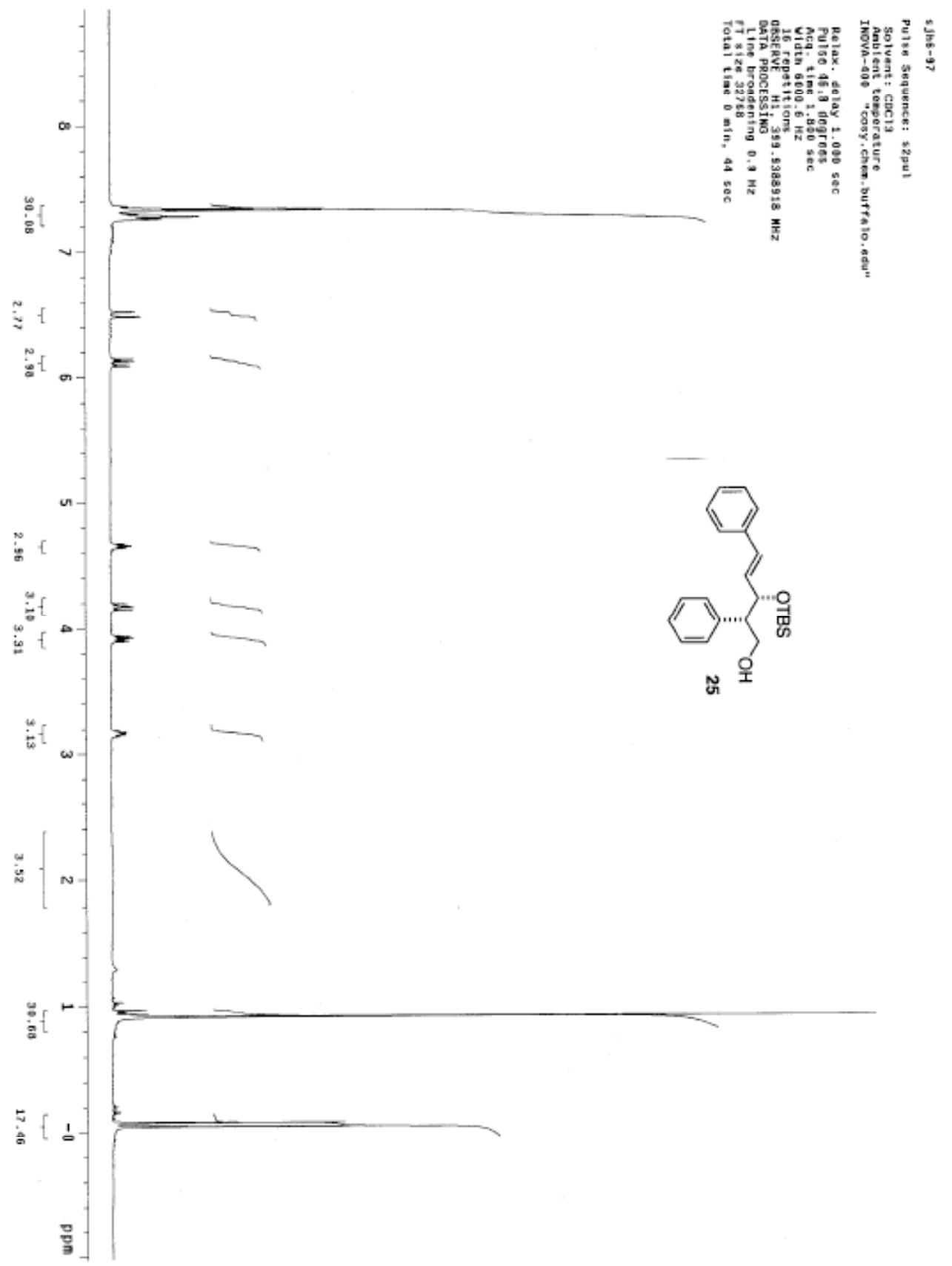




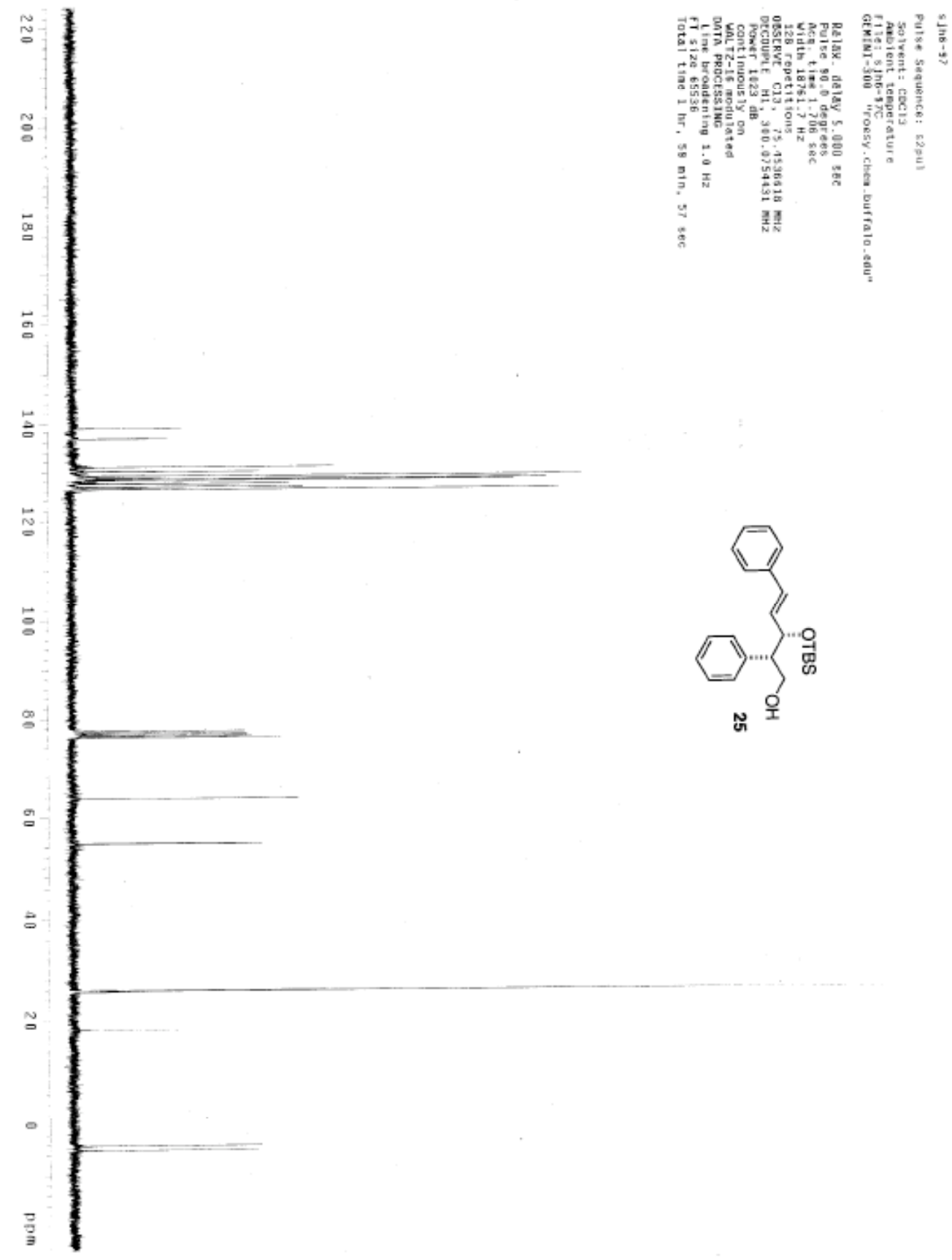


X-Ray Structure of (1R,2S)-2-(4-Bromophenyl)-1-(4-methoxyphenyl)propane-1,3diol (23)

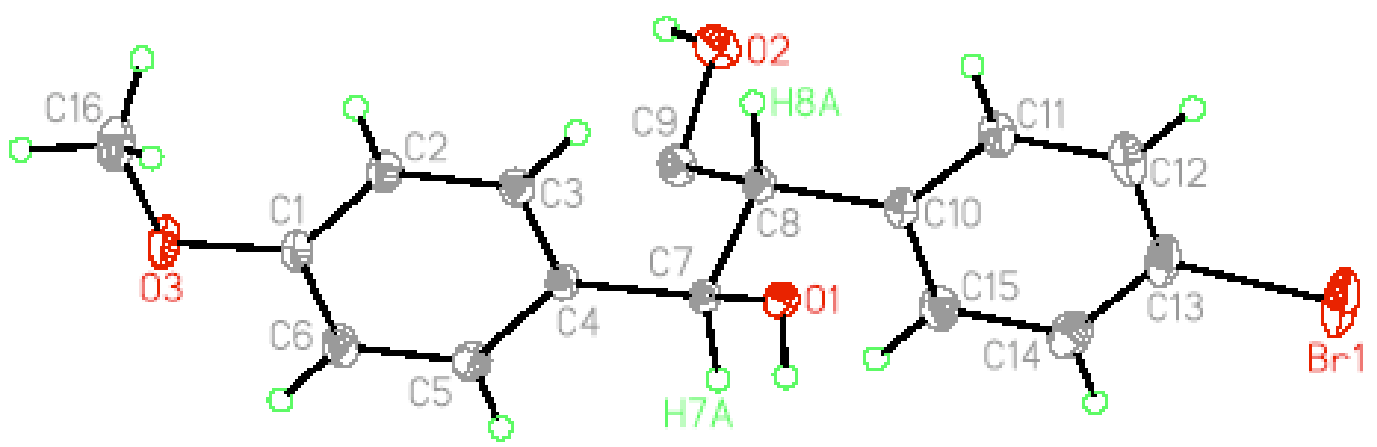

Figure S2. Geometry of the substituted ethylene glycol molecule (23) at $90 \mathrm{~K} .50 \%$ probability ellipsoids. The crystal structure has been deposited at the Cambridge Crystallographic Data Center and the deposition number CCDC 277546 has been allocated. $^{17}$

${ }^{17}$ Gerlits, O. O.; Coppens, P. Private Communication. 